\title{
A Generalization of the Secant Zeta Function as a Lambert Series
}

\author{
H.-Y. Li $\mathbb{D}^{1},{ }^{1}$ B. Maji, ${ }^{2}$ and T. Kuzumaki $\mathbb{D I}^{3}$ \\ ${ }^{1}$ Sanmenxia Suda Transportation Energy Saving Technology Co., Ltd., Sanmenxia, 472000 Henan, China \\ ${ }^{2}$ Discipline of Mathematics, Indian Institute of Technology Indore, Simrol, Indore 453552, Madhya Pradesh, India \\ ${ }^{3}$ Faculty of Engineering, Gifu University, Gifu 501-1193, Japan \\ Correspondence should be addressed to T. Kuzumaki; kuzumaki@gifu-u.ac.jp
}

Received 14 January 2020; Accepted 5 February 2020; Published 30 April 2020

Guest Editor: Praveen Agarwal

Copyright (c) 2020 H.-Y. Li et al. This is an open access article distributed under the Creative Commons Attribution License, which permits unrestricted use, distribution, and reproduction in any medium, provided the original work is properly cited.

Recently, Lalín, Rodrigue, and Rogers have studied the secant zeta function and its convergence. They found many interesting values of the secant zeta function at some particular quadratic irrational numbers. They also gave modular transformation properties of the secant zeta function. In this paper, we generalized secant zeta function as a Lambert series and proved a result for the Lambert series, from which the main result of Lalín et al. follows as a corollary, using the theory of generalized Dedekind etafunction, developed by Lewittes, Berndt, and Arakawa.

\section{Introduction}

The Dedekind eta-function and its limiting values have been considered by several authors starting from Riemann's posthumous fragment [1] and Wintner [2] and later by Reyna [3] and Wang [4]. There are many generalizations of the Dedekind eta-function as a Lambert series including those of Lewittes [5], Berndt [6], and Arakawa [7, 8]. In particular cases, they reduce to the cotangent or the cosecant zeta function. Lerch [9] in 1904 introduced the cotangent zeta function for an algebraic irrational number $z$ and an odd positive integer $s$ as

$$
\xi(z, s):=\sum_{n=1}^{\infty} \frac{\cot (n \pi z)}{n^{s}} .
$$

He stated the following functional equation for the cotangent zeta function, but without proof.

Theorem 1 (see [9]). For any algebraic irrational number $z$ and sufficiently large positive integer $k=k(z)$, we have

$$
\xi(z, 2 k+1)+z^{2 k} \xi\left(\frac{1}{z}, 2 k+1\right)=(2 \pi)^{2 k+1} \phi(z, 2 k+1)
$$

where

$$
\phi(z, n):=\sum_{i=0}^{n+1} \frac{B_{i} B_{n+1-j}}{j !(n+1-j) !} z^{j-1}
$$

where $B_{i}$ is the $i$-th Bernoulli number.

Berndt [10], in 1973, focused on the cotangent zeta function for general $s \in \mathbb{C}$ and proved Lerch's functional equation for cotangent zeta function. He found many interesting explicit formulae for $\xi(z, s)$ when $z$ is a quadratic irrational and $s \geq 3$ is an odd integer. One such pleasing formula is

$$
\xi\left(\frac{1+\sqrt{5}}{2}, 3\right)=-\frac{\pi^{3}}{45 \sqrt{5}} .
$$

In fact, Berndt's work implies that $\sqrt{j} \xi(\sqrt{j}, s) \pi^{-s} \in \mathbb{Q}$, where $j$ is any positive integer and $s \geq 3$ is an odd integer.

\section{Secant Zeta Function}

Recently, Lalín et al. [11] considered the secant zeta function

$$
\psi(z, s):=\sum_{n=1}^{\infty} \frac{\sec (n \pi z)}{n^{s}}
$$

and found its special values at some particular quadratic irrational arguments. They proved the following results. 
Theorem 2 (see [11], Theorem 1). The series (5) is absolutely convergent in the following cases:

(1) When $z=p / q$ is a rational number with $q$ odd and $s>1$.

(2) When $z$ is an algebraic irrational number and $s \geq 2$.

To prove this theorem, they have used the celebrated Thue-Siegel-Roth theorem.

Theorem 3 (see [11], Theorem 3). Let $E_{m}$ denote the Euler numbers and let $B_{m}$ denote the Bernoulli numbers. Suppose that $l$ is an even positive integer. Then, for appropriate values of $\alpha$,

$$
\begin{aligned}
& (\alpha+1)^{l-1} \psi\left(\frac{\alpha}{\alpha+1}, l\right)-(-\alpha+1)^{l-1} \psi\left(\frac{\alpha}{-\alpha+1}, l\right) \\
& =\frac{(\pi i)^{l}}{l !} \sum_{n=0}^{l}\left(2^{n-1}-1\right) B_{n} E_{l-n}\left(\begin{array}{l}
l \\
n
\end{array}\right)\left[(1+\alpha)^{n-1}-(1-\alpha)^{n-1}\right] .
\end{aligned}
$$

They found the values of the secant zeta function at some quadratic irrational numbers. For $j \in \mathbb{Z}$,

$$
\begin{aligned}
& \psi(\sqrt{2 j(2 j+1)}, 2)=(3 j+1) \frac{\pi^{2}}{6}, \\
& \psi(\sqrt{8 j(2 j+1)}, 2)=\frac{\pi^{2}}{6}, \\
& \psi(\sqrt{2 j(2 j+1)}, 4)=\frac{75 j^{2}+46 j+6}{8 j+3} \frac{\pi^{4}}{180} .
\end{aligned}
$$

After observing these values, they conjectured the following.

Conjecture 1 (see [11], Conjecture 1). If $j$ is any positive integer and $s$ is an even positive integer, then

$$
\psi(\sqrt{j}, s) \pi^{-s} \in \mathbb{Q} .
$$

By a clever use of residue theorem, Berndt and Straub [12] proved the above functional equation (6), and from it they derived

$$
\psi(\sqrt{r}, s) \pi^{-s} \in \mathbb{Q}, \quad r \in \mathbb{Q}^{+}, s \in 2 \mathbb{N} .
$$

Furthermore, they connected the secant Dirichlet series with Eichler integrals of Eisenstein series and checked unimodularity of period polynomials. On the contrary, Charollais and Greenberg [13] related the secant Dirichlet series $\psi(\alpha, s)$ to the generalized eta-function which was studied by Arakawa [7]. They proved that for $s \in 2 \mathbb{N}$,

$$
\psi(\alpha, s) \pi^{-s} \in \mathbb{Q}(\alpha),
$$

for all real quadratic irrationals $\alpha$. They used Arakawa's result to give an explicit formula for $\psi(\alpha, s)$ for real quadratic irrational numbers $\alpha$.
We will introduce a generalization of the secant zeta function as a Lambert series. Using the theory of generalized Dedekind eta-function due to Lewittes [5], Berndt [6], and Arakawa [7], we shall give a generalization of Theorem 3 .

We begin by briefly describing the theory of generalized Dedekind eta-function, developed by Lewittes [5], Berndt [6], and Arakawa [7], which is a main tool in our study.

\section{Work of Lewittes and Berndt}

Lewittes and Berndt treat the case of the upper half-plane $\mathbb{\square}$ while Arakawa treats the case of upper half plane limiting to an algebraic irrational number. Hereafter, we use the following notations:

$$
\begin{aligned}
e[w]:=\exp (2 \pi i w), \quad w \in \mathbb{C}, \\
\langle x\rangle \in \mathbb{R}, \quad 0<\langle x\rangle \leqq 1, \quad x-\langle x\rangle \in \mathbb{Z}, \\
\{x\} \in \mathbb{R}, \quad 0 \leqq\{x\}<1, \quad x-\{x\} \in \mathbb{Z} .
\end{aligned}
$$

Lewittes [5] defined the generalization of the Dedekind eta-function as a Lambert series. For a pair $\left(r_{1}, r_{2}\right)$ of real numbers, $z \in \mathbb{W}$ and arbitrary $s \in \mathbb{C}$, he considered the series

$$
A\left(z, s, r_{1}, r_{2}\right):=\sum_{m>-r_{1}} \sum_{k=1}^{\infty} k^{s-1} e\left[k r_{2}+k\left(m+r_{1}\right) z\right],
$$

where the first summation is over all integers $m$ with $m>-r_{1}$. He also introduced its associate as

$$
H\left(z, s, r_{1}, r_{2}\right):=A\left(z, s, r_{1}, r_{2}\right)+e\left[\frac{s}{2}\right] A\left(z, s,-r_{1},-r_{2}\right) .
$$

Let $s=r_{1}=r_{2}=0$. Put $A(z, 0,0,0)=A(z)$, then $H(z, 0,0,0)=2 A(z)$. Using the product definition of Dedekind eta-function $\eta(z)$, it is easy to show that

$$
\log (\eta(z))=\frac{\pi i}{12}-A(z) .
$$

Let us see a couple of examples.

Example 1. For special choices of parameters $r_{1}$ and $r_{2}$, the A- and $H$-functions reduce to the cosecant and cotangent zeta functions:

$$
\begin{aligned}
\frac{1}{(1+e[s / 2])} H\left(z, s,\left(\frac{1}{2}, 0\right)\right) & =A\left(z, s, \frac{1}{2}, 0\right) \\
& =\sum_{m>-(1 / 2)} \sum_{k=1}^{\infty} k^{s-1} e\left[k\left(m+\frac{1}{2}\right) z\right] \\
& =\sum_{k=1}^{\infty} k^{s-1} \frac{e[(1 / 2) k z]}{1-e[k z]} \\
& =\frac{i}{2} \sum_{k=1}^{\infty} \frac{\operatorname{cosec}(\pi k z)}{k^{1-s}} .
\end{aligned}
$$


Also,

$$
\begin{aligned}
\frac{1}{(1+e[s / 2])} H(z, s,(1,0)) & =A(z, s, 1,0) \\
& =\sum_{m>-1} \sum_{k=1}^{\infty} k^{s-1} e[k(m+1) z] \\
& =\sum_{k=1}^{\infty} k^{s-1} \frac{e[k z]}{1-e[k z]} \\
& =\frac{1}{2} \sum_{k=1}^{\infty} k^{s-1}\left\{\frac{1+e[k z]}{1-e[k z]}-1\right\} \\
& =\frac{i}{2} \sum_{k=1}^{\infty} \frac{\cot (\pi k z)}{k^{1-s}}-\frac{1}{2} \zeta(1-s) .
\end{aligned}
$$

Some more definitions will be required.

Definition 1 (Hurwitz zeta function). For a positive number a, the Hurwitz zeta function

$$
\begin{aligned}
\zeta(s, a):=\sum_{n=0}^{\infty}(n+a)^{-s}, \\
\\
\Re(s)>1 .
\end{aligned}
$$

Definition 2. Let $\Omega$ denote the characteristic function of integers, i.e.,

$$
\Omega(a):= \begin{cases}1, & a \in \mathbb{Z} \\ 0, & a \notin \mathbb{Z} .\end{cases}
$$

For any positive number $\lambda$, let $I(\lambda, \infty)$ denote the integration path consisting of the oriented line segment $(+\infty, \lambda)$, the positively oriented circle of radius $\lambda$ with center at the origin, and the oriented line segment $(\lambda,+\infty)$.

Let

$$
G_{2}\left(z,\left(\omega_{1}, \omega_{2}\right) ; t\right):=\frac{\exp (-z t)}{\left(1-\exp \left(-\omega_{1} t\right)\right)\left(1-\exp \left(-\omega_{2} t\right)\right)},
$$

for any pair $\left(\omega_{1}, \omega_{2}\right)$ of positive numbers and for $z, t \in \mathbb{C}$.

Berndt [6] proved the following transformation formula.

Theorem 4 (see [6], Theorem 2). Let $V=\left(\begin{array}{ll}a & b \\ c & d\end{array}\right) \epsilon$ $S L(2, \mathbb{Z})$ with $c>0$. For any pair $\left(r_{1}, r_{2}\right)$ of real numbers, set $R_{1}=r_{1} a+r_{2} c, R_{2}=r_{1} b+r_{2} d, \rho=\left\{R_{2}\right\} c-\left\{R_{1}\right\} d$. For $z \in \mathbb{U}$ with $c \Re(z)+d>0$, let $\beta=c z+d$. Then, for arbitrary $s \in \mathbb{C}$, we have

$$
\begin{aligned}
\beta^{-s} H\left(V z, s, r_{1}, r_{2}\right)-H\left(z, s, R_{1}, R_{2}\right) \\
=-\Omega\left(r_{1}\right)(2 \pi)^{-s} e\left[\frac{s}{4}\right] \beta^{-s} \Gamma(s)\left(\zeta\left(s,\left\langle r_{2}\right\rangle\right)+e\left[\frac{s}{2}\right] \zeta\left(s,\left\langle-r_{2}\right\rangle\right)\right) \\
\quad+\Omega\left(R_{1}\right)(2 \pi)^{-s} e\left[-\frac{s}{4}\right] \Gamma(s)\left(\zeta\left(s,\left\langle-R_{2}\right\rangle\right)+e\left[\frac{s}{2}\right] \zeta\left(s,\left\langle R_{2}\right\rangle\right)\right) \\
\quad+(2 \pi)^{-s} e\left[-\frac{s}{4}\right] L\left(z, s, R_{1}, R_{2}, c, d\right),
\end{aligned}
$$

where

$$
\begin{aligned}
& L\left(z, s, R_{1}, R_{2}, c, d\right) \\
& =-\sum_{j=1}^{c} \int_{I(\lambda, \infty)} t^{s-1} \frac{\exp \left(-\left(1-\{(j d+\varrho) / c\}+\left((c z+d)\left(j-\left\{R_{1}\right\}\right)\right) / c\right) t\right)}{(1-\exp (-t))(1-\exp (-(c z+d) t))} \mathrm{d} t, \quad 0<\lambda<2 \pi, \frac{2 \pi}{|\beta|} .
\end{aligned}
$$

Here, $\log t$ is understood to be real-valued on the upper segment $(+\infty, \lambda)$ of $I(\lambda, \infty)$.

\section{Work of Arakawa}

Arakawa studied certain Lambert series associated to a complex variable $s$ and an irrational real algebraic number $\alpha$. Those Lambert series are defined as limiting (boundary) values of the generalized Dedekind eta-functions studied by Berndt [6]. Arakawa obtained transformation formulae under the action of $\operatorname{SL}(2, \mathbb{Z})$ on those $\alpha$.

For an irrational real algebraic number $\alpha$ and a pair $(p, q)$ of real numbers, Arakawa [7] introduced a generalized eta-function defined as

$$
\eta(\alpha, s, p, q):=\sum_{n=1}^{\infty} n^{s-1} \frac{e[n(p \alpha+q)]}{1-e[n \alpha]}, \quad s \in \mathbb{C},
$$

and its associate by

$$
H(\alpha, s,(p, q)):=\eta(\alpha, s,\langle p\rangle, q)+e\left[\frac{s}{2}\right] \eta(\alpha, s,\langle-p\rangle,-q) .
$$

Example 2. Again, if we consider $(p, q)=(1 / 2,0)$ and $(p, q)=(1,0)$, then also we will get the cosecant and cotangent zeta function: 


$$
\begin{aligned}
\frac{1}{(1+e[s / 2])} H\left(\alpha, s,\left(\frac{1}{2}, 0\right)\right) & =\eta\left(\alpha, s, \frac{1}{2}, 0\right) \\
& =\sum_{k=1}^{\infty} k^{s-1} \frac{e[(1 / 2) k \alpha]}{1-e[k \alpha]} \\
& =\frac{i}{2} \sum_{k=1}^{\infty} \frac{\operatorname{cosec}(\pi k \alpha)}{k^{1-s}}, \\
\frac{1}{(1+e[s / 2])} H(\alpha, s,(1,0))= & \eta(\alpha, s, 1,0) \\
& =\sum_{k=1}^{\infty} k^{s-1} \frac{e[k z]}{1-e[k z]} \\
& =\frac{i}{2} \sum_{k=1}^{\infty} \frac{\cot (\pi k \alpha)}{k^{1-s}}-\frac{1}{2} \zeta(1-s),
\end{aligned}
$$

where $s \in \mathbb{C}$ with $\mathfrak{R}(s)<0$.

Theorem 5 (see [7], Lemma 1 and Theorem 2). Suppose $\alpha \in \mathbb{R} \cap \overline{\mathbb{Q}}$ and $\alpha \notin \mathbb{Q}$. Then, the infinite series $\eta(\alpha, s, p, q)$ is absolutely convergent if $\Re(s)<0$. If, in addition, $[\mathbb{Q}(\alpha): \mathbb{Q}]=2$ and $(p, q) \in \mathbb{Q}^{2}$, then $H(\alpha, s, p, q)$ has analytic continuation to $\mathbb{C}-\{0\}$, and the singularity at $s=0$ is at worst a simple pole.

Arakawa proved the absolute convergence of $\eta(\alpha, s, p, q)$ for $\mathfrak{R}(s)<0$, by using the Thue-Siegel-Roth theorem.

Consider the generalized eta-function

$$
\eta(z, s, p, q)=\sum_{n=1}^{\infty} n^{s-1} \frac{e[n(p z+q)]}{1-e[n z]}, \quad s \in \mathbb{C}
$$

corresponding to (22), for $z \in \mathbb{H}$ and a pair $(p, q) \in \mathbb{R}^{2}$ with $p>0$. Then, one can see that this series is absolutely convergent for arbitrary $s \in \mathbb{C}$. It can be easily checked that there is a link between the infinite series $A\left(z, s, r_{1}, r_{2}\right)$ and $\eta\left(z, s, r_{1}, r_{2}\right)$.

Lemma 1. For any pair $\left(r_{1}, r_{2}\right) \in \mathbb{R}^{2}$ and $z \in \mathbb{H}$, we have

$$
A\left(z, s, r_{1}, r_{2}\right)=\eta\left(z, s,\left\langle r_{1}\right\rangle, r_{2}\right), \quad s \in \mathbb{C} \text {. }
$$

Now, from the definition of $H$-function (13), we have

$$
H\left(z, s, r_{1}, r_{2}\right)=A\left(z, s, r_{1}, r_{2}\right)+e\left[\frac{s}{2}\right] A\left(z, s,-r_{1},-r_{2}\right) \text {. }
$$

Hence, using Lemma 1, we get

$H\left(z, s, r_{1}, r_{2}\right)=\eta\left(z, s,\left\langle r_{1}\right\rangle, r_{2}\right)+e\left[\frac{s}{2}\right] \eta\left(z, s,\left\langle-r_{1}\right\rangle,-r_{2}\right)$.

Similarly, we have
Lemma 2. For any algebraic irrational number $\alpha$ and a pair $(p, q) \in \mathbb{R}^{2}$,

$$
A(\alpha, s, p, q)=\eta(\alpha, s,\langle p\rangle, q), \quad \mathfrak{R}(s)<0 .
$$

Again by the definition of $H$-function (23)(due to Arakawa), we have

$$
H(\alpha, s, p, q)=\eta(\alpha, s,\langle p\rangle, q)+e\left[\frac{s}{2}\right] \eta(\alpha, s,\langle-p\rangle, q) .
$$

Therefore, by Lemma 2, we get

$$
H(\alpha, s, p, q)=A(\alpha, s, p, q)+e\left[\frac{s}{2}\right] A(\alpha, s,-p, q) .
$$

Proposition 1 (see [7], Proposition 1). Let $V=\left(\begin{array}{ll}a & b \\ c & d\end{array}\right) \in S L(2, \mathbb{Z}), \alpha$ be an irrational real algebraic number, and $(p, q) \in \mathbb{R}^{2}$ with $p>0$. Let $z=\alpha+i y$ with $y>0$. Set $z^{*}=V z$ and $\beta=V \alpha=(a \alpha+b)(c \alpha+d)^{-1}$. If $\mathfrak{R}(s)<-3$, then

$$
\lim _{y \longrightarrow 0^{+}} \eta\left(z^{*}, s, p, q\right)=\eta(\beta, s, p, q) .
$$

Arakawa obtained the following transformation formulae for $H(\alpha, s,(p, q))$, by virtue of Theorem 4 of Berndt and Proposition 1.

Theorem 6 (see [7], Theorem 1). Let $\alpha$ be any real algebraic irrational, and let $V=\left(\begin{array}{ll}a & b \\ c & d\end{array}\right) \in \operatorname{SL}(2, \mathbb{Z})$ with $c>0$ such that $\beta:=c \alpha+d>0$. For any pair $(p, q)$ of real numbers, set $p^{\prime}=p a+q c, q^{\prime}=p b+q d$, and $\rho=\left\{q^{\prime}\right\} c-\left\{p^{\prime}\right\} d$. Then, for $\mathfrak{R}(s)<0$,

$$
\begin{aligned}
D_{1}(V, \alpha, s,(p, q)):= & \beta^{-s} H(V \alpha, s,(p, q))-H(\alpha, s,(p, q) V) \\
= & \beta^{-s} H(V \alpha, s,(p, q))-H\left(\alpha, s,\left(p^{\prime}, q^{\prime}\right)\right) \\
= & -\Omega(p)(2 \pi)^{-s} e\left[\frac{s}{4}\right] \beta^{-s} \Gamma(s) \\
& \cdot\left(\zeta(s,\langle q\rangle)+e\left[\frac{s}{2}\right] \zeta(s,\langle-q\rangle)\right) \\
& +\Omega\left(p^{\prime}\right)(2 \pi)^{-s} e\left[-\frac{s}{4}\right] \Gamma(s) \\
& \cdot\left(\zeta\left(s,\left\langle-q^{\prime}\right\rangle\right)+e\left[\frac{s}{2}\right] \zeta\left(s,\left\langle q^{\prime}\right\rangle\right)\right) \\
& +(2 \pi)^{-s} e\left[-\frac{s}{4}\right] L\left(\alpha, s,\left(p^{\prime}, q^{\prime}\right), c, d\right),
\end{aligned}
$$

where 


$$
L\left(\alpha, s, p^{\prime}, q^{\prime}, c, d\right)=-\sum_{j=1}^{c} \int_{I(\lambda, \infty)} t^{s-1} G_{2}\left(1-\left\{\frac{(j d+\rho)}{c}\right\}+\frac{\left(j-\left\{p^{\prime}\right\}\right) \beta}{c},(1, \beta) ; t\right) \mathrm{d} t, \quad 0<\lambda<2 \pi, \frac{2 \pi}{\beta} .
$$

Berndt [6] (p. 499) found the special values of $L(\alpha, s$, $\left.\left(p^{\prime}, q^{\prime}\right), c, d\right)$ at nonnegative integral arguments $s=-m$ :

$$
\begin{aligned}
L\left(\alpha,-m,\left(p^{\prime}, q^{\prime}\right), c, d\right)= & \frac{2 \pi i}{(m+2) !} \sum_{j=1}^{c} \sum_{k=0}^{m+2}\left(\begin{array}{c}
m+2 \\
k
\end{array}\right) \\
& \cdot B_{k}\left(\frac{j-\left\{p^{\prime}\right\}}{c}\right) \bar{B}_{m+2-k}\left(\frac{j d+\rho}{c}\right) \\
& \cdot(-\beta)^{k-1},
\end{aligned}
$$

where $B_{n}(x)$ denotes the $n$th Bernoulli polynomial and $\bar{B}_{n}(x)=B_{n}(\{x\})$.

Lemma 3 (see [7], Lemma 4). Let $\alpha$ be an irrational number in a real quadratic field $\mathbb{Q}(\Delta)$ and let $(p, q)$ be a pair of rational numbers. Then, there exist a totally positive unit $\beta$ of $\mathbb{Q}(\Delta)$ and an element $V=\left(\begin{array}{ll}a & b \\ c & d\end{array}\right)$ of $S L(2, \mathbb{Z})$ which satisfy
the conditions:

$$
\begin{aligned}
& \text { (i) } c>0 \\
& \text { (ii) }(p, q) V \equiv(p, q) \bmod 1 \\
& \text { (iii) } \beta\left(\begin{array}{l}
\alpha \\
1
\end{array}\right)=V\left(\begin{array}{l}
\alpha \\
1
\end{array}\right)
\end{aligned}
$$

We choose such $\beta \in \mathbb{Q}(\Delta)$ and $V \in S L(2, \mathbb{Z})$, i.e., which satisfy the conditions of Lemma 3. Then, using condition (ii), we have

$$
H(\alpha, s,(p, q))=H(\alpha, s,(p, q) V) .
$$
that

Since $V \alpha=\alpha$ and $c>0$, we can see easily from Theorem 6

$$
\begin{aligned}
H(\alpha, s,(p, q))= & -\Omega(p)(2 \pi)^{-s} e\left[\frac{s}{4}\right] \Gamma(s) \zeta(s,\langle q\rangle) \\
& +\Omega(p)(2 \pi)^{-s} e\left[-\frac{s}{4}\right] \Gamma(s) \zeta(s,\langle q\rangle) \\
& \cdot \frac{1-e[s] \beta^{-s}}{\beta^{-s}-1}+\frac{(2 \pi)^{-s} e[-(s / 4)]}{\beta^{-s}-1} \\
& \cdot L(\alpha, s,(p, q), c, d) .
\end{aligned}
$$

Example 3. Let $\alpha, \beta$, and $V$ as in Lemma 3 and with $(p, q)=$ $(1,0)$ and $(p, q)=(1 / 2,0)$. Then,

$$
\begin{aligned}
H(\alpha, s,(1,0))= & (2 \pi)^{-s}\left(-e\left[\frac{s}{4}\right]+e\left[-\frac{s}{4}\right] \frac{1-e[s] \beta^{-s}}{\beta^{-s}-1}\right) \Gamma(s) \zeta(s) \\
& +\frac{(2 \pi)^{-s} e[-(s / 4)]}{\beta^{-s}-1} L(\alpha, s,(1,0), c, d), \\
H\left(\alpha, s,\left(\frac{1}{2}, 0\right)\right)= & \frac{(2 \pi)^{-s} e[-(s / 4)]}{\beta^{-s}-1} L\left(\alpha, s,\left(\frac{1}{2}, 0\right), c, d\right) .
\end{aligned}
$$

Values at some particular matrices. Let

$$
\begin{aligned}
& V_{0}=\left(\begin{array}{cc}
0 & -1 \\
1 & 0
\end{array}\right), \\
& V_{1}=\left(\begin{array}{ll}
1 & 0 \\
1 & 1
\end{array}\right), \\
& V_{2}=V_{0}^{2} V_{1}^{-1}=\left(\begin{array}{cc}
-1 & 0 \\
1 & -1
\end{array}\right) .
\end{aligned}
$$

Example 4. Theorem 6 gives the following:

$$
\begin{aligned}
D_{1}\left(V_{0}, \alpha, s,(p, q)\right)= & \alpha^{-s} H\left(\frac{-1}{\alpha}, s,(p, q)\right)-H(\alpha, s,(q,-p)) \\
= & -\Omega(p)(2 \pi)^{-s} e\left[\frac{s}{4}\right] \alpha^{-s} \Gamma(s) \\
& \cdot\left(\zeta(s,\langle q\rangle)+e\left[\frac{s}{2}\right] \zeta(s,\langle-q\rangle)\right) \\
& +\Omega(q)(2 \pi)^{-s} e\left[-\frac{s}{4}\right] \Gamma(s) \\
& \cdot\left(\zeta(s,\langle p\rangle)+e\left[\frac{s}{2}\right] \zeta(s,\langle-p\rangle)\right) \\
& +(2 \pi)^{-s} e\left[-\frac{s}{4}\right] L(\alpha, s,(q,-p), 1,0), \\
D_{1}\left(V_{1}, \alpha, s,(p, q)\right)= & (\alpha+1)^{-s} H\left(\frac{\alpha}{\alpha+1}, s,(p, q)\right) \\
& -H(\alpha, s,(p+q, q)) \\
= & -\Omega(p)(2 \pi)^{-s} e\left[\frac{s}{4}\right](\alpha+1)^{-s} \Gamma(s) \\
& \cdot\left(\zeta(s,\langle q\rangle)+e\left[\frac{s}{2}\right] \zeta(s,\langle-q\rangle)\right) \\
& +\Omega(p+q)(2 \pi)^{-s} e\left[-\frac{s}{4}\right] \Gamma(s) \\
& \cdot\left(\zeta(s,\langle-q\rangle)+e\left[\frac{s}{2}\right] \zeta(s,\langle q\rangle)\right) \\
& +(2 \pi)^{-s} e\left[-\frac{s}{4}\right] L(\alpha, s,(p+q, q), 1,1),
\end{aligned}
$$

$$
\begin{aligned}
D_{1}\left(V_{2}, \alpha, s,(p, q)\right)= & (\alpha-1)^{-s} H\left(\frac{-\alpha}{\alpha-1}, s,(p, q)\right) \\
& -H(\alpha, s,(-p+q,-q)) \\
= & -\Omega(p)(2 \pi)^{-s} e\left[\frac{s}{4}\right](\alpha-1)^{-s} \Gamma(s) \\
& \cdot\left(\zeta(s,\langle q\rangle)+e\left[\frac{s}{2}\right] \zeta(s,\langle-q\rangle)\right) \\
& +\Omega(-p+q)(2 \pi)^{-s} e\left[-\frac{s}{4}\right] \Gamma(s) \\
& \cdot\left(\zeta(s,\langle q\rangle)+e\left[\frac{s}{2}\right] \zeta(s,\langle-q\rangle)\right) \\
& +(2 \pi)^{-s} e\left[-\frac{s}{4}\right] L(\alpha, s,(-p+q,-q), 1,-1) .
\end{aligned}
$$


In particular, when $(p, q)=(1,0)$, we have

$$
\begin{aligned}
& D_{1}\left(V_{0}, \alpha, s,(1,0)\right)=(2 \pi)^{-s} e\left[\frac{s}{4}\right]\left\{e\left[-\frac{s}{2}\right]-\alpha^{-s}\right\} \Gamma(s)\left(1+e\left[\frac{s}{2}\right]\right) \zeta(s)+(2 \pi)^{-s} e\left[-\frac{s}{4}\right] L(\alpha, s,(0,-1), 1,0), \\
& D_{1}\left(V_{1}, \alpha, s,(1,0)\right)=(2 \pi)^{-s} e\left[\frac{s}{4}\right]\left\{e\left[-\frac{s}{2}\right]-(\alpha+1)^{-s}\right\} \Gamma(s)\left(1+e\left[\frac{s}{2}\right]\right) \zeta(s)+(2 \pi)^{-s} e\left[-\frac{s}{4}\right] L(\alpha, s,(1,0), 1,1), \\
& D_{1}\left(V_{2}, \alpha, s,(1,0)\right)=(2 \pi)^{-s} e\left[\frac{s}{4}\right]\left\{e\left[-\frac{s}{2}\right]-(\alpha-1)^{-s}\right\} \Gamma(s)\left(1+e\left[\frac{s}{2}\right]\right) \zeta(s)+(2 \pi)^{-s} e\left[-\frac{s}{4}\right] L(\alpha, s,(-1,0), 1,-1) .
\end{aligned}
$$

If we choose $(p, q)=(1 / 2,0)$, we get

$$
\begin{aligned}
D_{1}\left(V_{0}, \alpha, s,\left(\frac{1}{2}, 0\right)\right) & =\alpha^{-s} H\left(\frac{-1}{\alpha}, s,\left(\frac{1}{2}, 0\right)\right)-H\left(\alpha, s,\left(0 .-\frac{1}{2}\right)\right) \\
& =(2 \pi)^{-s}\left(e\left[\frac{s}{4}\right]+e\left[-\frac{s}{4}\right]\right) \Gamma(s) \zeta\left(s, \frac{1}{2}\right)+(2 \pi)^{-s} e\left[-\frac{s}{4}\right] L\left(\alpha, s,\left(0,-\frac{1}{2}\right), 1,0\right) \\
D_{1}\left(V_{1}, \alpha, s,\left(\frac{1}{2}, 0\right)\right) & =(\alpha+1)^{-s} H\left(\frac{\alpha}{\alpha+1}, s,\left(\frac{1}{2}, 0\right)\right)-H\left(\alpha, s,\left(\frac{1}{2}, 0\right)\right) \\
& =(2 \pi)^{-s} e\left[-\frac{s}{4}\right] L\left(\alpha, s,\left(\frac{1}{2}, 0\right), 1,1\right) \\
D_{1}\left(V_{2}, \alpha, s,\left(\frac{1}{2}, 0\right)\right) & =(\alpha-1)^{-s} H\left(\frac{-\alpha}{\alpha-1}, s,\left(\frac{1}{2}, 0\right)\right)-H\left(\alpha, s,\left(-\frac{1}{2}, 0\right)\right) \\
& =(2 \pi)^{-s} e\left[-\frac{s}{4}\right] L\left(\alpha, s,\left(-\frac{1}{2}, 0\right), 1,-1\right) .
\end{aligned}
$$

Note that for nonnegative integers $m$, we have the following explicit formulae for $V_{j}$, where $j=0,1,2$ :

$$
\begin{aligned}
L\left(\alpha,-m,(1,0) V_{j}, c, d\right)= & \frac{2 \pi i}{(m+2) !} \sum_{k=0}^{m+2}\left(\begin{array}{c}
m+2 \\
k
\end{array}\right) \\
& \cdot B_{k}(1) \bar{B}_{m+2-k}(1)(-\beta)^{k-1}, \\
L\left(\alpha,-m,\left(\frac{1}{2}, 0\right) V_{j}, c, d\right)= & \frac{2 \pi i}{(m+2) !} \sum_{k=0}^{m+2}\left(\begin{array}{c}
m+2 \\
k
\end{array}\right) \\
& \cdot B_{k}\left(\frac{1}{2}\right) \bar{B}_{m+2-k}\left(\frac{1}{2}\right)(-\beta)^{k-1} .
\end{aligned}
$$

\section{Generalization of the Secant Zeta Function}

We introduce two Lambert series corresponding to (22) and (12). These include the generalizations of secant and tangent zeta functions as shown in Example 5. Let $\alpha$ be any algebraic irrational number and $(p, q)$ a pair of real numbers. Then, we define the series $\eta^{*}$ by

$$
\eta^{*}(\alpha, s, p, q):=\sum_{n=1}^{\infty} n^{s-1} \frac{e[n(p \alpha+q)]}{1+e[n \alpha]}, \quad \mathfrak{R}(s)<0
$$

and another infinite series $A^{*}$ by

$$
A^{*}\left(z, s, r_{1}, r_{2}\right):=\sum_{m>-r_{1}}(-1)^{m} \sum_{k=1}^{\infty} k^{s-1} e\left[k r_{2}+k\left(m+r_{1}\right) z\right] \text {, }
$$

for a pair $\left(r_{1}, r_{2}\right) \in \mathbb{R}^{2}, z \in \mathbb{H}$, and $s \in \mathbb{C}$.

Example 5. If we take $\left(r_{1}, r_{2}\right)=(1,0)$, and $(1 / 2,0)$, then (48) becomes

$$
\begin{aligned}
A^{*}(\alpha, s, 1,0) & =\eta^{*}(\alpha, s, 1,0) \\
& =\sum_{k=1}^{\infty} k^{s-1} \frac{e[k \alpha]}{1+e[k \alpha]} \\
& =\frac{1}{2} \sum_{k=1}^{\infty} k^{s-1}\left(\frac{e[k \alpha]-1}{1+e[k \alpha]}+1\right) \\
& =\frac{i}{2} \sum_{k=1}^{\infty} k^{s-1} \tan (\pi k \alpha)+\frac{1}{2} \zeta(1-s),
\end{aligned}
$$




$$
\begin{aligned}
A^{*}\left(\alpha, s, \frac{1}{2}, 0\right) & =\eta\left(\alpha, s, \frac{1}{2}, 0\right) \\
& =\frac{1}{2} \sum_{k=1}^{\infty} k^{s-1} \frac{1}{\cos (\pi k \alpha)} \\
& =\frac{1}{2} \psi(\alpha, 1-s),
\end{aligned}
$$

respectively.

By virtue of the results of Lewittes, Berndt, and Arakawa, we have the following results.

Lemma 4. Let $\alpha$ be an algebraic irrational number and $(p, q)$ be a pair of real numbers. The series $\eta^{*}(\alpha, s, p, q)$ is absolutely convergent, if $s \in \mathbb{C}$ with $\mathfrak{R}(s)<0$.

Proof. One can prove this result applying the Thue-Siegel-Roth theorem, in a similar manner to Arakawa's procedure for proving the absolute convergence of the series $\eta(\alpha, s, p, q)$.

Lemma 5. If $z \in \mathbb{H}$ and a pair $(p, q) \in \mathbb{R}^{2}$ with $p>0$, then the series $\eta^{*}(z, s, p, q)$ is absolutely convergent for any $s \in \mathbb{C}$.

Proof. Since $z \in \mathbb{H}$, assume $z=x+i y$ with $y>0$. We have

$$
\begin{aligned}
\left|\eta^{*}(z, s, p, q)\right| & =\left|\sum_{n=1}^{\infty} n^{s-1} \frac{e[n(p z+q)]}{1+e[n z]}\right| \\
& \leqq \sum_{n=1}^{\infty} n^{\sigma-1} \frac{\exp (-2 \pi n p y)}{1-\exp (-2 \pi n y)},
\end{aligned}
$$

for $\Re(s)=\sigma .1-\exp (-2 \pi n y) \geqq 1-\exp (-2 \pi y)$. And we can choose a large enough positive integer $K$ such that for $n>K$ $n^{\sigma-1} \exp (-2 \pi n p y)=\exp ((\sigma-1) \log n-2 \pi n p y) \leqq \exp (-\pi n p y)$.

Thus,

$$
\begin{aligned}
\left|\eta^{*}(z, s, p, q)\right| & \leq \sum_{n=1}^{\infty} n^{\sigma-1} \frac{\exp (-2 \pi n p y)}{1-\exp (-2 \pi n y)} \\
& \leq \sum_{n=1}^{K} n^{\sigma-1} \frac{\exp (-2 \pi n p y)}{1-\exp (-2 \pi n y)}+\sum_{n=K+1}^{\infty} n^{\sigma-1} \frac{\exp (-2 \pi n p y)}{1-\exp (-2 \pi y)} \\
& \leq \sum_{n=1}^{K} n^{\sigma-1} \frac{\exp (-2 \pi n p y)}{1-\exp (-2 \pi n y)}+\sum_{n=K+1}^{\infty} \frac{\exp (-\pi n p y)}{1-\exp (-2 \pi y)} \\
& \leq \sum_{n=1}^{K} n^{\sigma-1} \frac{\exp (-2 \pi n p y)}{1-\exp (-2 \pi n y)} \\
& +\frac{\exp (-\pi(K+1) p y)}{1-\exp (-2 \pi p y)} \frac{1}{1-\exp (-2 \pi y)}<\infty .
\end{aligned}
$$

Lemma 6. Let $z \in \mathbb{H}$ and $\alpha$ be an irrational algebraic number. Then, for any pair of real numbers $\left(r_{1}, r_{2}\right)$, we have

$$
\begin{array}{ll}
A^{*}\left(z, s, r_{1}, r_{2}\right)=(-1)^{-r_{1} \nless\left\langle r_{1}\right\rangle} \eta^{*}\left(z, s,\left\langle r_{1}\right\rangle, r_{2}\right), & s \in \mathbb{C}, \\
A^{*}\left(\alpha, s, r_{1}, r_{2}\right)=(-1)^{-r_{1} \nless\left\langle r_{1}\right\rangle} \eta^{*}\left(\alpha, s,\left\langle r_{1}\right\rangle, r_{2}\right), & \mathfrak{R}(s)<0 .
\end{array}
$$

Proof. If $r_{1} \in \mathbb{Z}$, then $m>-r_{1}$ implies $m=-r_{1}+r$ for $r=1,2,3, \ldots$, . By the definition of $A^{*}\left(z, s, r_{1}, r_{2}\right)$, we know

$$
\begin{aligned}
A^{*}\left(z, s, r_{1}, r_{2}\right) & =\sum_{m>-r_{1}}(-1)^{m} \sum_{k=1}^{\infty} k^{s-1} e\left[k r_{2}+k\left(m+r_{1}\right) z\right] \\
& =\sum_{k=1}^{\infty} k^{s-1} \sum_{r=1}^{\infty}(-1)^{-r_{1}+r} e\left[k r_{2}+k r z\right] \\
& =(-1)^{-r_{1}+1} \sum_{k=1}^{\infty} k^{s-1} e\left[k r_{2}+k z\right] \sum_{r=0}^{\infty}(-1)^{r} e[k r z] \\
& =(-1)^{-r_{1}+1} \sum_{k=1}^{\infty} k^{s-1} \frac{e\left[k r_{2}+k z\right]}{1+e[k z]} \\
& =(-1)^{\left.-r_{1} \nless r_{1}\right\rangle} \eta^{*}\left(z, s,\left\langle r_{1}\right\rangle, r_{2}\right),
\end{aligned}
$$

since $\left\langle r_{1}\right\rangle=1$.

Again, if $r_{1} \notin \mathbb{Z}, m>-r_{1}$ implies $m=-\left\lfloor r_{1}\right\rfloor+r$ for $r=0,1,2, \ldots$, . So, we will have

$$
\begin{aligned}
A^{*}\left(z, s, r_{1}, r_{2}\right) & =\sum_{m>-r_{1}}(-1)^{m} \sum_{k=1}^{\infty} k^{s-1} e\left[k r_{2}+k\left(m+r_{1}\right) z\right] \\
& =\sum_{k=1}^{\infty} k^{s-1} \sum_{r=0}^{\infty}(-1)^{\left\lfloor r_{1}\right\rfloor+r} e\left[k r_{2}+k\left(\left\langle r_{1}\right\rangle+r\right) z\right] \\
& =(-1)^{-\left\lfloor r_{1}\right\rfloor} \sum_{k=1}^{\infty} k^{s-1} e\left[k r_{2}+k\left\langle r_{1}\right\rangle z\right] \sum_{r=0}^{\infty}(-1)^{r} e[k r z] \\
& =(-1)^{-\left\lfloor r_{1}\right\rfloor} \sum_{k=1}^{\infty} k^{s-1} \frac{e\left[k r_{2}+k\left\langle r_{1}\right\rangle z\right]}{1+e[k z]} \\
& =(-1)^{-r_{1} \nmid\left\langle r_{1}\right\rangle} \eta^{*}\left(z, s,\left\langle r_{1}\right\rangle, r_{2}\right) .
\end{aligned}
$$

Similarly, we can see that

$A^{*}\left(\alpha, s, r_{1}, r_{2}\right)=(-1)^{-r_{1}+\left\langle r_{1}\right\rangle} \eta^{*}\left(\alpha, s,\left\langle r_{1}\right\rangle, r_{2}\right), \quad$ for $\mathfrak{R}(s)<0$.

Lemma 7. If $z \in \mathbb{H}, A^{*}\left(z, s, r_{1}, r_{2}\right)$ is absolutely convergent for any $s \in \mathbb{C}$.

Proof. Using Lemmas 5 and 6, we can show that $A^{*}\left(z, s, r_{1}, r_{2}\right)$ is absolutely convergent for $s \in \mathbb{C}$. 


\section{Main Results}

Consider the difference

$$
\begin{aligned}
D^{*}(V):= & D^{*}\left(V, \alpha, s, \frac{1}{2}, 0\right):=\beta^{-s} A^{*}\left(V \alpha, s, \frac{1}{2}, 0\right) \\
& -A^{*}\left(\alpha, s, \frac{1}{2}, 0\right),
\end{aligned}
$$

for each $V$ from (40). Now, the second term in the above expression is the secant zeta function in view of (50). This difference is quite natural in the sense that it expresses the surplus after the modular transformation is applied.
We interpret the main result of Lalín et al. Theorem 3 in this setting as a special case of

$$
(\alpha+1)^{-s} A^{*}\left(V_{1} \alpha, s, \frac{1}{2}, 0\right)+(\alpha-1)^{-s} A^{*}\left(V_{2} \alpha, s, \frac{1}{2}, 0\right),
$$

for $\mathfrak{R}(s)<0$, and locate it in a natural way as we will see in Corollary 1 . Our main theorem is the following.

Theorem 7. For a real algebraic irrational $\alpha$ and a complex variable $s$ with $\mathfrak{R}(s)<0$, we have

$$
\begin{aligned}
D^{*}\left(V_{0}\right)= & \alpha^{-s} A^{*}\left(\frac{-1}{\alpha}, s, \frac{1}{2}, 0\right)-A^{*}\left(\alpha, s, \frac{1}{2}, 0\right) \\
= & 2^{1-2 s} \pi^{-s} e\left[-\frac{s}{4}\right]\left(\Phi_{0}+\Gamma(s) \Omega_{0}\right)+2^{1-s} \Psi_{0} \\
= & -\frac{(2 \pi)^{-s} e[-s / 4]}{1-e[s / 2]} \int_{I(\lambda, \infty)} t^{s-1} \frac{\exp (-(1 / 2+\alpha) t)}{(1+\exp (-t))(1-\exp (-\alpha t))} \mathrm{d} t+2^{-2 s} \pi^{-s} e\left[-\frac{s}{4}\right] \Gamma(s)\left(\zeta\left(s, \frac{1}{4}\right)-\zeta\left(s, \frac{3}{4}\right)\right) \\
& -2^{1-s} \sum_{n=1}^{\infty} n^{s-1} \frac{e[n(\alpha / 2+1 / 4)](e[n \alpha / 2]+1)}{1-e[n \alpha]} 2^{2-s} \sum_{n=1}^{\infty}(2 n)^{s-1} \frac{e[3 \pi \alpha / 2]}{1-e[2 n \alpha]}, \\
D^{*}\left(V_{1}\right)= & (\alpha+1)^{-s} A^{*}\left(\frac{\alpha}{\alpha+1}, s, \frac{1}{2}, 0\right)-A^{*}\left(\alpha, s, \frac{1}{2}, 0\right) \\
= & 2^{1-2 s} \pi^{-s} e\left[-\frac{s}{4}\right] \Phi_{1}+2^{1-s} \Psi_{1} \\
= & -\frac{(2 \pi)^{-s} e[-s / 4]}{1-e[s / 2]} \int_{I(\lambda, \infty)} t^{s-1} \frac{\exp (-t / 2)}{(1+\exp (-t))} \frac{\exp (-(\alpha+1) t / 2)}{(1-\exp (-(\alpha+1) t))} \mathrm{d} t+2^{-s} \sum_{n=1}^{\infty} n^{s-1} \frac{(-1)^{n-1}}{\cos (\pi n \alpha / 2)} .
\end{aligned}
$$

Also,

$$
\begin{aligned}
D^{*}\left(V_{2}\right) & =(\alpha-1)^{-s} A^{*}\left(\frac{-\alpha}{\alpha-1}, s, \frac{1}{2}, 0\right)-A^{*}\left(\alpha, s, \frac{1}{2}, 0\right) \\
& =2^{1-2 s} \pi^{-s} e\left[-\frac{s}{4}\right] \Phi_{2}+2^{1-s} \Psi_{2} \\
& =-\frac{(2 \pi)^{-s} e[-s / 4]}{1-e[s / 2]} \int_{I(\lambda, \infty)} t^{s-1} \frac{\exp (-t / 2)}{(1+\exp (-t))} \frac{\exp (-(\alpha-1) t / 2)}{(1-\exp (-(\alpha-1) t))} \mathrm{d} t-2^{-s} \sum_{n=1}^{\infty} n^{s-1} \frac{1}{\cos (\pi n \alpha / 2)},
\end{aligned}
$$

where $\Phi_{k}$ and $\Psi_{k}$ and $(k=0,1,2)$ are defined later. They indicate the block of L-integrals and the block of H-functions, corresponding to the matrix $V_{k}$, respectively. Also, $\Omega_{0}$ is defined in (90).
We recover the main result of Lalin et al. ([11], Theorem 3), i.e., Theorem 3 by adding the equations (61) and (62). We note it as a corollary. 


\section{Corollary 1.}

$$
\begin{aligned}
& (\alpha+1)^{-s} A^{*}\left(\frac{\alpha}{\alpha+1}, s, \frac{1}{2}, 0\right)+(\alpha-1)^{-s} A^{*}\left(\frac{-\alpha}{\alpha-1}, s, \frac{1}{2}, 0\right) \\
& =-\frac{(2 \pi)^{-s} e[-s / 4]}{1-e[s / 2]} \int_{I(\lambda, \infty)} t^{s-1} \sum_{m=0}^{\infty} 2^{-m-1} E_{m} \frac{t^{m}}{m !} \sum_{n=0}^{\infty}\left(2^{1-n}-1\right) B_{n} \\
& \quad \times \frac{\left\{(\alpha+1)^{n-1}+(\alpha-1)^{n-1}\right\} t^{n-1}}{n !} \mathrm{d} t .
\end{aligned}
$$

The genesis of the transformation formula of Lalín et al. ([11], Theorem 3 ) for the secant zeta function is given by the sum of $D^{*}\left(V_{1}\right)$ and $D^{*}\left(V_{2}\right)$, which we have seen in Corollary 1 . We will see in the proof of Corollary 1 that the term $2 A^{*}(\alpha, s, 1 / 2,0)$ on the left side and the secant zeta function on the right hand side naturally cancel each other. As this occurs only in such a pairing, this elucidates the hidden structure of the paired transformation formula from a more general standpoint.

Deduction of the Main Theorem of Lalin et al. Firstly, we deduce Theorem 3 from Corollary 1 . To do that, let $l=2 k$ be an even positive integer and $s=1-l$. Then, (63) amounts to

$$
\begin{aligned}
(\alpha & +1)^{2 k-1} A^{*}\left(\frac{\alpha}{\alpha+1},-2 k+1, \frac{1}{2}, 0\right)+(\alpha-1)^{2 k-1} A^{*}\left(\frac{-\alpha}{\alpha-1},-2 k+1, \frac{1}{2}, 0\right) \\
& =-\frac{(2 \pi)^{2 k-1} e[-((-2 k+1) / 4)]}{1-e[(-2 k+1) / 2]} \int_{I(\lambda, \infty)} t^{-2 k} \sum_{m=0}^{\infty} 2^{-m-1} E_{m} \frac{t^{m}}{m !} \sum_{n=0}^{\infty}\left(2^{1-n}-1\right) B_{n} \frac{\left\{(\alpha+1)^{n-1}+(\alpha-1)^{n-1}\right\} t^{n-1}}{n !} \mathrm{d} t \\
& =-\frac{2^{2 k-1} \pi^{2 k}(-1)^{k}}{2 \pi i} \int_{I(\lambda, \infty)} t^{-2 k} \sum_{m=0}^{\infty} \sum_{n=0}^{\infty} 2^{-m-1}\left(2^{1-n}-1\right) E_{m} B_{n}\left\{(\alpha+1)^{n-1}+(\alpha-1)^{n-1}\right\} \frac{t^{m+n-1}}{m ! n !} \mathrm{d} t \\
& =-2^{2 k-1} \pi^{2 k}(-1)^{k} \sum_{n=0}^{2 k} \frac{1}{(2 k-n) ! n !} 2^{-2 k+n-1}\left(2^{1-n}-1\right) E_{2 k-n} B_{n}\left\{(\alpha+1)^{n-1}+(\alpha-1)^{n-1}\right\} \\
& =\frac{1}{2} \pi^{2 k}(-1)^{k} \sum_{n=0}^{2 k} \frac{1}{(2 k-n) ! n !}\left(2^{n-1}-1\right) E_{2 k-n} B_{n}\left\{(\alpha+1)^{n-1}+(\alpha-1)^{n-1}\right\} .
\end{aligned}
$$

This proves Theorem 3 .

The following conjecture seems to be plausible.

Conjecture 2. Let $W_{1}=\left(\begin{array}{ll}a_{1} & b_{1} \\ c_{1} & d_{1}\end{array}\right)$ and $W_{2}=\left(\begin{array}{ll}a_{2} & b_{2} \\ c_{2} & d_{2}\end{array}\right)$ be two matrices in $P S L_{2}(\mathbb{Z})$ which are inverses to each other. Then, for a pair $(p, q) \in \mathbb{R}^{2}$,

$$
\left(c_{1} \alpha+d_{1}\right)^{-s} A^{*}\left(W_{1} \alpha, s, p, q\right)+\left(c_{2} \alpha+d_{2}\right)^{-s} A^{*}\left(W_{2} \alpha, s, p, q\right)
$$

can be expressible in terms of special values of the zeta and Lfunctions as we have seen for the sum of two explicit expressions for

$$
\left(c_{1} \alpha+d_{1}\right)^{-s} A^{*}\left(V_{j} \alpha, s,\left(\frac{1}{2}, 0\right)\right)-A^{*}\left(\alpha, s,\left(\frac{1}{2}, 0\right)\right), \quad j=1,2 .
$$

\section{7. $A^{*}$ in Terms of $A$ - and $H$-Functions}

Before proving our main theorem we need to express $A^{*}$ in terms of $A$ and $H$. We know that given a sum $S=\sum_{n} a_{n}$ with its even and odd parts $S_{e}$ and $S_{o}$, where the even part is over all even integer values and odd part over odd integer values, the sum $2 S_{e}-S$ is the alternating sum $\sum_{n}(-1)^{n} a_{n}$. Using this observation, we have the following result.
Lemma 8. $A^{*}\left(z, s, r_{1}, r_{2}\right)=2 A\left(2 z, s, r_{1} / 2, r_{2}\right)-A\left(z, s, r_{1}, r_{2}\right)$.

Proof. By the definition of $A^{*}\left(z, s, r_{1}, r_{2}\right)$, we have

$$
\begin{aligned}
A^{*}\left(z, s, r_{1}, r_{2}\right)= & \sum_{m>-r_{1}}(-1)^{m} \sum_{k=1}^{\infty} k^{s-1} e\left[k r_{2}+k\left(m+r_{1}\right) z\right] \\
= & 2 \sum_{\substack{m>-r_{1} \\
m: \text { even }}} \sum_{\substack{\infty \\
\text { s. }}}^{\infty} k^{s-1} e\left[k r_{2}+k\left(m+r_{1}\right) z\right] \\
& -\sum_{m>-r_{1}} \sum_{k=1}^{\infty} k^{s-1} e\left[k r_{2}+k\left(m+r_{1}\right) z\right] \\
= & 2 \sum_{2 m>-r_{1}} \sum_{k=1}^{\infty} k^{s-1} e\left[k r_{2}+k\left(2 m+r_{1}\right) z\right] \\
& -\sum_{m>-r_{1}} \sum_{k=1}^{\infty} k^{s-1} e\left[k r_{2}+k\left(m+r_{1}\right) z\right] \\
= & 2 A\left(2 z, s, \frac{r_{1}}{2}, r_{2}\right)-A\left(z, s, r_{1}, r_{2}\right) .
\end{aligned}
$$


There is a duplication formula for $A\left(z, s, r_{1}, r_{2}\right)$ which is as follows:

\section{Lemma}

$2^{s} A\left(2 z, s, r_{1}, 2 r_{2}\right)$.

Proof. From Definition 1 of $A\left(z, s, r_{1}, r_{2}\right)$, we have

$$
\begin{aligned}
A & \left(z, s, r_{1}, r_{2}\right)+A\left(z, s, r_{1}, r_{2}+\frac{1}{2}\right) \\
= & \sum_{m>-r_{1}} \sum_{k=1}^{\infty} k^{s-1} e\left[k r_{2}+k\left(m+r_{1}\right) z\right] \\
& +\sum_{m>-r_{1}} \sum_{k=1}^{\infty} k^{s-1} e\left[k\left(r_{2}+\frac{1}{2}\right)+k\left(m+r_{1}\right) z\right] \\
= & \sum_{m>-r_{1}} \sum_{k=1}^{\infty} k^{s-1} e\left[k r_{2}+k\left(m+r_{1}\right) z\right]\left(1+e\left[\frac{1}{2} k\right]\right) \\
= & 2 \sum_{m>-r_{1}} \sum_{k=1}^{\infty}(2 k)^{s-1} e\left[2 k r_{2}+2 k\left(m+r_{1}\right) z\right] \\
= & 2^{s} \sum_{m>-r_{1}} \sum_{k=1}^{\infty} k^{s-1} e\left[k\left(2 r_{2}\right)+k\left(m+r_{1}\right)(2 z)\right] \\
= & 2^{s} A\left(2 z, s, r_{1}, 2 r_{2}\right) .
\end{aligned}
$$

Using the duplication formula, i.e., Lemma 9 in Lemma 8 , we get

\section{Lemma 10.}

$$
\begin{aligned}
A^{*}\left(z, s, r_{1}, r_{2}\right)= & 2^{1-s} A\left(z, s, \frac{r_{1}}{2}, \frac{r_{2}}{2}\right)+2^{1-s} A\left(z, s, \frac{r_{1}}{2}, \frac{r_{2}}{2}+\frac{1}{2}\right) \\
& -A\left(z, s, r_{1}, r_{2}\right) .
\end{aligned}
$$

On the other hand,

$$
\begin{array}{r}
H\left(z, s, r_{1}, r_{2}\right)=A\left(z, s, r_{1}, r_{2}\right)+e\left[\frac{s}{2}\right] A\left(z, s,-r_{1},-r_{2}\right), \\
H\left(z, s,-r_{1},-r_{2}\right)=A\left(z, s,-r_{1},-r_{2}\right)+e\left[\frac{s}{2}\right] A\left(z, s, r_{1}, r_{2}\right), \\
H\left(z, s,-r_{1},-r_{2}\right)=A\left(z, s,-r_{1},-r_{2}\right)+e\left[\frac{s}{2}\right] A\left(z, s, r_{1}, r_{2}\right) .
\end{array}
$$

Therefore,

$$
\begin{aligned}
A\left(z, s, r_{1}, r_{2}\right)= & \frac{1}{1-e[s]}\left\{H\left(z, s, r_{1}, r_{2}\right)\right. \\
& \left.-e\left[\frac{s}{2}\right] H\left(z, s,-r_{1},-r_{2}\right)\right\} .
\end{aligned}
$$

Substituting (71) in Lemma 10, we deduce the following proposition.
Proposition 2. For a real algebraic irrational $\alpha$, a pair $(p, q)$ of real numbers with $p>0$, and a complex variable $s$ with $\mathfrak{R}(s)<0$, we have

$$
\begin{aligned}
(1-e[s]) A^{*}(\alpha, s, p, q)= & 2^{1-s}\left\{H\left(\alpha, s,\left(\frac{p}{2}, \frac{q}{2}\right)\right)\right. \\
& \left.-e\left[\frac{s}{2}\right] H\left(\alpha, s,\left(-\frac{p}{2},-\frac{q}{2}\right)\right)\right\} \\
& +2^{1-s}\left\{H\left(\alpha, s,\left(\frac{p}{2}, \frac{q}{2}+\frac{1}{2}\right)\right)\right. \\
& \left.-e\left[\frac{s}{2}\right] H\left(\alpha, s,\left(-\frac{p}{2},-\frac{q}{2}-\frac{1}{2}\right)\right)\right\} \\
& -(1-e[s]) A(\alpha, s, p, q),
\end{aligned}
$$

where

$$
\begin{aligned}
(1-e[s]) A(\alpha, s, p, q)= & \{H(\alpha, s,(p, q)) \\
& \left.-e\left[\frac{s}{2}\right] H(\alpha, s,(-p,-q))\right\},
\end{aligned}
$$

as in equation (71).

Example 6. If we consider $(p, q)=(1,0)$ and $(1 / 2,0)$, then we get

$$
\begin{aligned}
A^{*}(\alpha, s, 1,0)= & \frac{1}{1+e[s / 2]}\left\{2^{1-s} H\left(\alpha, s,\left(\frac{1}{2}, 0\right)\right)\right. \\
& +2^{1-s} H\left(\alpha, s,\left(\frac{1}{2}, \frac{1}{2}\right)\right)-H(\alpha, s, \\
A^{*}\left(\alpha, s, \frac{1}{2}, 0\right)= & \frac{2^{1-s}}{1-e[s]} H\left(\alpha, s,\left(\frac{1}{4}, 0\right)\right) \\
& -\frac{2^{1-s} e[s / 2]}{1-e[s]} H\left(\alpha, s,\left(-\frac{1}{4}, 0\right)\right) \\
& +\frac{2^{1-s}}{1-e[s]} H\left(\alpha, s,\left(\frac{1}{4}, \frac{1}{2}\right)\right) \\
& -\frac{2^{1-s} e[s / 2]}{1-e[s]} H\left(\alpha, s,\left(-\frac{1}{4},-\frac{1}{2}\right)\right) \\
& -\frac{1-e[s / 2]}{1-e[s]} H\left(\alpha, s,\left(\frac{1}{2}, 0\right)\right) .
\end{aligned}
$$$$
\left.+2^{1-s} H\left(\alpha, s,\left(\frac{1}{2}, \frac{1}{2}\right)\right)-H(\alpha, s,(1,0))\right\},
$$

For the last term, with $s$ an even integer, we use either

$$
H\left(\alpha, s,\left(\frac{1}{2}, 0\right)\right)=\frac{(2 \pi)^{-s} e[-s / 4]}{\beta^{-s}-1} L\left(\alpha, s,\left(\frac{1}{2}, 0\right), c, d\right)
$$

or

$$
\frac{1}{1+e[s / 2]} H\left(\alpha, s,\left(\frac{1}{2}, 0\right)\right)=\frac{i}{2} \sum_{k=1}^{\infty} \frac{1}{k^{1-s}} \frac{1}{\sin (\pi k \alpha)},
$$

which follows from Examples 1 and 3, respectively. 


\section{General Procedure}

The general procedure is to transform

$$
A\left(z, s, r_{1}, r_{2}\right)=\frac{1}{1-e[s]}\left\{H\left(z, s, r_{1}, r_{2}\right)-e\left[\frac{s}{2}\right] H\left(z, s,-r_{1},-r_{2}\right)\right\} .
$$

$D^{*}(V):=D^{*}(V, \alpha, s, p, q):=\beta^{-s} A^{*}(V \alpha, s, p, q)-A^{*}(\alpha, s, p, q)$.

Now using Proposition 2, we can write

We recall the following notations

$D_{1}(V, \alpha, s,(p, q))=\beta^{-s} H(V \alpha, s,(p, q))-H(\alpha, s,(p, q) V)$,

$D_{0}^{*}(V, \alpha, s, p, q)=\beta^{-s} A(V \alpha, s, p, q)-A(\alpha, s, p, q)$,

$$
\begin{aligned}
D^{*}(V, \alpha, s, p, q)+D_{0}^{*}(V, \alpha, s, p, q)= & \frac{2^{1-s}}{1-e[s]}\left(\beta^{-s} H\left(V \alpha, s,\left(\frac{p}{2}, \frac{q}{2}\right)\right)-H\left(\alpha, s,\left(\frac{p}{2}, \frac{q}{2}\right)\right)\right) \\
& -\frac{2^{1-s} e[s / 2]}{1-e[s]}\left(\beta^{-s} H\left(V \alpha, s,\left(-\frac{p}{2},-\frac{q}{2}\right)\right)-H\left(\alpha, s,\left(-\frac{p}{2},-\frac{q}{2}\right)\right)\right) \\
& +\frac{2^{1-s}}{1-e[s]}\left(\beta^{-s} H\left(V \alpha, s,\left(\frac{p}{2}, \frac{q}{2}+\frac{1}{2}\right)\right)-H\left(\alpha, s,\left(\frac{p}{2}, \frac{q}{2}+\frac{1}{2}\right)\right)\right) \\
& -\frac{2^{1-s} e[s / 2]}{1-e[s]}\left(\beta^{-s} H\left(V \alpha, s,\left(-\frac{p}{2},-\frac{q}{2}-\frac{1}{2}\right)\right)-H\left(\alpha, s,\left(-\frac{p}{2},-\frac{q}{2}-\frac{1}{2}\right)\right)\right) .
\end{aligned}
$$

For $(p, q)=(1 / 2,0)$, we have

$$
\begin{aligned}
D_{0}^{*}\left(V, \alpha, s, \frac{1}{2}, 0\right)= & \frac{1}{1+e[s / 2]}\left(\beta^{-s} H\left(V \alpha, s,\left(\frac{1}{2}, 0\right)\right)\right. \\
& \left.-H\left(\alpha, s,\left(\frac{1}{2}, 0\right)\right)\right) .
\end{aligned}
$$

$$
\begin{aligned}
D^{*}(V, \alpha, s, p, q)+D_{0}^{*}(V, \alpha, s, p, q)= & \frac{2^{1-s}}{1-e[s]}\left(D_{1}\left(V, \alpha, s,\left(\frac{p}{2}, \frac{q}{2}\right)\right)+H\left(\alpha, s,\left(\frac{p}{2}, \frac{q}{2}\right) V\right)-H\left(\alpha, s,\left(\frac{p}{2}, \frac{q}{2}\right)\right)\right) \\
& -\frac{2^{1-s} e[s / 2]}{1-e[s]}\left(D_{1}\left(V, \alpha, s,\left(-\frac{p}{2},-\frac{q}{2}\right)\right)+H\left(\alpha, s,\left(-\frac{p}{2},-\frac{q}{2}\right) V\right)-H\left(\alpha, s,\left(-\frac{p}{2},-\frac{q}{2}\right)\right)\right) \\
& +\frac{2^{1-s}}{1-e[s]}\left(D_{1}\left(V, \alpha, s,\left(\frac{p}{2}, \frac{q}{2}+\frac{1}{2}\right)\right)+H\left(\alpha, s,\left(\frac{p}{2}, \frac{q}{2}+\frac{1}{2}\right) V\right)-H\left(\alpha, s,\left(\frac{p}{2}, \frac{q}{2}+\frac{1}{2}\right)\right)\right) \\
& -\frac{2^{1-s} e[s / 2]}{1-e[s]}\left(D_{1}\left(V, \alpha, s,\left(-\frac{p}{2},-\frac{q}{2}-\frac{1}{2}\right)\right)+H\left(\alpha, s,\left(-\frac{p}{2},-\frac{q}{2}-\frac{1}{2}\right) V\right)-H\left(\alpha, s,\left(-\frac{p}{2},-\frac{q}{2}-\frac{1}{2}\right)\right)\right),
\end{aligned}
$$

where

$$
\begin{aligned}
D_{0}^{*}(V, \alpha, s, p, q)= & \frac{1}{1-e[s]}\left(D_{1}(V, \alpha, s,(p, q))\right. \\
& +H(\alpha, s,(p, q) V)-H(\alpha, s,(p, q))) \\
& -\frac{e[s / 2]}{1-e[s]}\left(D_{1}(V, \alpha, s,(-p,-q))\right. \\
& +H(\alpha, s,(-p,-q) V)-H(\alpha, s,(-p,-q))),
\end{aligned}
$$

in the case of (79), while

$$
\begin{aligned}
D_{0}^{*}\left(V, \alpha, s, \frac{1}{2}, 0\right)= & \frac{1}{1+e[s / 2]}\left(D_{1}\left(V, \alpha, s,\left(\frac{1}{2}, 0\right)\right)\right. \\
& \left.+H\left(\alpha, s,\left(\frac{1}{2}, 0\right) V\right)-H\left(\alpha, s,\left(\frac{1}{2}, 0\right)\right)\right),
\end{aligned}
$$

(84)

in the case of (82). Hence, 


$$
\begin{aligned}
D^{*}(V, \alpha, s, p, q)+D_{0}^{*}(V, \alpha, s, p, q)= & \frac{2^{1-s}}{1-e[s]} D_{1}\left(V, \alpha, s,\left(\frac{p}{2}, \frac{q}{2}\right)\right)-\frac{2^{1-s} e[s / 2]}{1-e[s]} D_{1}\left(V, \alpha, s,\left(-\frac{p}{2},-\frac{q}{2}\right)\right) \\
& +\frac{2^{1-s}}{1-e[s]} D_{1}\left(V, \alpha, s,\left(\frac{p}{2}, \frac{q}{2}+\frac{1}{2}\right)\right)-\frac{2^{1-s} e[s / 2]}{1-e[s]} D_{1}\left(V, \alpha, s,\left(-\frac{p}{2},-\frac{q}{2}-\frac{1}{2}\right)\right) \\
& +\frac{2^{1-s}}{1-e[s]}\left(H\left(\alpha, s,\left(\frac{p}{2}, \frac{q}{2}\right) V\right)-H\left(\alpha, s,\left(\frac{p}{2}, \frac{q}{2}\right)\right)\right) \\
& -\frac{2^{1-s} e[s / 2]}{1-e[s]}\left(H\left(\alpha, s,\left(-\frac{p}{2},-\frac{q}{2}\right) V\right)-H\left(\alpha, s,\left(-\frac{p}{2},-\frac{q}{2}\right)\right)\right)+\frac{2^{1-s}}{1-e[s]}\left(H\left(\alpha, s,\left(\frac{p}{2}, \frac{q}{2}+\frac{1}{2}\right) V\right)\right. \\
& \left.-H\left(\alpha, s,\left(\frac{p}{2}, \frac{q}{2}+\frac{1}{2}\right)\right)\right) \\
& -\frac{2^{1-s} e[s / 2]}{1-e[s]}\left(H\left(\alpha, s,\left(-\frac{p}{2},-\frac{q}{2}-\frac{1}{2}\right) V\right)-H\left(\alpha, s,\left(-\frac{p}{2},-\frac{q}{2}-\frac{1}{2}\right)\right)\right),
\end{aligned}
$$

where the last term is either (79) or (85).

$$
\begin{aligned}
D^{*}\left(V_{0}\right)= & D^{*}\left(V_{0}, \alpha, s, \frac{1}{2}, 0\right)=\alpha^{-s} A^{*}\left(\frac{-1}{\alpha}, s, \frac{1}{2}, 0\right)-A^{*}\left(\alpha, s, \frac{1}{2}, 0\right) \\
= & \frac{2^{1-s}}{1-e[s]} D_{1}\left(V_{0}, \alpha, s,\left(\frac{1}{4}, 0\right)\right)+\frac{2^{1-s} e[s / 2]}{1-e[s]} D_{1}\left(V_{0}, \alpha, s,\left(-\frac{1}{4}, 0\right)\right) \\
& +\frac{2^{1-s}}{1-e[s]} D_{1}\left(V_{0}, \alpha, s,\left(\frac{1}{4}, \frac{1}{2}\right)\right)-\frac{2^{1-s} e[s / 2]}{1-e[s]} D_{1}\left(V_{0}, \alpha, s,\left(-\frac{1}{4},-\frac{1}{2}\right)\right) \\
& -\frac{1-e[s / 2]}{1-e[s]} D_{1}\left(V_{0}, \alpha, s,\left(\frac{1}{2}, 0\right)\right)+\frac{2^{1-s}}{1-e[s]}\left(H\left(\alpha, s,\left(\frac{1}{4}, 0\right) V_{0}\right)-H\left(\alpha, s,\left(\frac{1}{4}, 0\right)\right)\right) \\
& -\frac{2^{1-s}}{1-e[s]}\left\{H\left(\alpha, s,\left(\frac{1}{4}, 0\right) V_{0}\right)-H\left(\alpha, s,\left(-\frac{1}{4}, 0\right)\right)\right\} \\
& +\frac{2^{1-s}}{1-e[s]}\left\{H\left(\alpha, s,\left(\frac{1}{4}, \frac{1}{2}\right) V_{0}\right)-H\left(\alpha, s,\left(\frac{1}{4}, \frac{1}{2}\right)\right)\right\} \\
& -\frac{1-e[s / 2]}{1-e[s]}\left\{H\left(\alpha, s,\left(\frac{1}{2}, 0\right) V_{0}\right)-H\left(\alpha, s,\left(\frac{1}{2}, 0\right)\right)\right\} . \\
& \frac{2^{1-s} e[s / 2]}{1-e[s]}\left\{H\left(\alpha, s,\left(-\frac{1}{4},-\frac{1}{2}\right) V_{0}\right)-H\left(\alpha, s,\left(-\frac{1}{4},-\frac{1}{2}\right)\right)\right\}
\end{aligned}
$$


Then, applying (41), we deduce that

$$
\begin{aligned}
D^{*}\left(V_{0}\right)= & \frac{2^{1-s}}{1-e[s]}(2 \pi)^{-s}\left\{e\left[-\frac{s}{4}\right] L\left(\alpha, s,\left(0,-\frac{1}{4}\right), 1,0\right)-e\left[\frac{s}{4}\right] L\left(\alpha, s,\left(0, \frac{1}{4}\right), 1,0\right)\right\} \\
& +\frac{2^{1-s}}{1-e[s]}(2 \pi)^{-s}\left\{e\left[-\frac{s}{4}\right] L\left(\alpha, s,\left(\frac{1}{2},-\frac{1}{4}\right), 1,0\right)-e\left[\frac{s}{4}\right] L\left(\alpha, s,\left(-\frac{1}{2}, \frac{1}{4}\right), 1,0\right)\right\} \\
& -\frac{1-e[s / 2]}{1-e[s]}(2 \pi)^{-s} e\left[-\frac{s}{4}\right] L\left(\alpha, s,\left(0,-\frac{1}{2}\right), 1,0\right)-\frac{2^{1-s} e[s / 2]}{1-e[s]}(2 \pi)^{-s} e\left[-\frac{s}{4}\right] \Gamma(s)\left(\zeta\left(s, \frac{3}{4}\right)+e\left[\frac{s}{2}\right] \zeta\left(s, \frac{1}{4}\right)\right) \\
& +\frac{2^{1-s}}{1-e[s]}(2 \pi)^{-s} e\left[-\frac{s}{4}\right] \Gamma(s)\left(\zeta\left(s, \frac{1}{4}\right)+e\left[\frac{s}{2}\right] \zeta\left(s, \frac{3}{4}\right)\right)-\frac{1-e[s / 2]}{1-e[s]}(2 \pi)^{-s} e\left[-\frac{s}{4}\right] \Gamma(s)\left(\zeta\left(s, \frac{1}{2}\right)+e\left[\frac{s}{2}\right] \zeta\left(s, \frac{1}{2}\right)\right) \\
& -\frac{1-e[s / 2]}{1-e[s]}\left\{H\left(\alpha, s,\left(0,-\frac{1}{2}\right)\right)-H\left(\alpha, s,\left(\frac{1}{2}, 0\right)\right)\right\}+\frac{2^{1-s}}{1-e[s]}\left\{H\left(\alpha, s,\left(0,-\frac{1}{4}\right)\right)-H\left(\alpha, s,\left(\frac{1}{4}, 0\right)\right)\right\} \\
& -\frac{2^{1-s}[s / 2]}{1-e[s]}\left\{H\left(\alpha, s,\left(0, \frac{1}{4}\right)\right)-H\left(\alpha, s,\left(-\frac{1}{4}, 0\right)\right)\right\}+\frac{2^{1-s}}{1-e[s]}\left\{H\left(\alpha, s,\left(\frac{1}{2},-\frac{1}{4}\right)\right)-H\left(\alpha, s,\left(\frac{1}{4}, \frac{1}{2}\right)\right)\right\} \\
& -\frac{2^{1-s} e[s / 2]}{1-e[s]}\left\{H\left(\alpha, s,\left(-\frac{1}{2}, \frac{1}{4}\right)\right)-H\left(\alpha, s,\left(-\frac{1}{4},-\frac{1}{2}\right)\right)\right\} .
\end{aligned}
$$

Let

$$
\begin{aligned}
(1-e[s]) \Phi_{0}= & L\left(\alpha, s,\left(0,-\frac{1}{4}\right), 1,0\right)+L\left(\alpha, s,\left(\frac{1}{2},-\frac{1}{4}\right), 1,0\right)-e\left[\frac{s}{2}\right] L\left(\alpha, s,\left(0, \frac{1}{4}\right), 1,0\right)-e\left[\frac{s}{2}\right] L\left(\alpha, s,\left(-\frac{1}{2}, \frac{1}{4}\right), 1,0\right) \\
& -\left(1-e\left[\frac{s}{2}\right]\right) 2^{s-1} L\left(\alpha, s,\left(0,-\frac{1}{2}\right), 1,0\right), \\
(1-e[s]) \Omega_{0}= & \zeta\left(s, \frac{1}{4}\right)-e[s] \zeta\left(s, \frac{1}{4}\right)-2^{s-1}\left(1-e\left[\frac{s}{2}\right]\right)\left(\zeta\left(s, \frac{1}{2}\right)+e\left[\frac{s}{2}\right] \zeta\left(s, \frac{1}{2}\right)\right), \\
(1-e[s]) \Psi_{0}= & H\left(\alpha, s,\left(0,-\frac{1}{4}\right)\right)-H\left(\alpha, s,\left(\frac{1}{4}, 0\right)\right)-e\left[\frac{s}{2}\right] H\left(\alpha, s,\left(0, \frac{1}{4}\right)\right) \\
& +e\left[\frac{s}{2}\right] H\left(\alpha, s,\left(-\frac{1}{4}, 0\right)\right)+H\left(\alpha, s,\left(\frac{1}{2},-\frac{1}{4}\right)\right)-H\left(\alpha, s,\left(\frac{1}{4}, \frac{1}{2}\right)\right) \\
& -e\left[\frac{s}{2}\right] H\left(\alpha, s,\left(-\frac{1}{2}, \frac{1}{4}\right)\right)+e\left[\frac{s}{2}\right] H\left(\alpha, s,\left(-\frac{1}{4},-\frac{1}{2}\right)\right) \\
& -2^{s-1}\left(1-e\left[\frac{s}{2}\right]\right)\left\{H\left(\alpha, s,\left(0,-\frac{1}{2}\right)\right)-H\left(\alpha, s,\left(\frac{1}{2}, 0\right)\right)\right\} .
\end{aligned}
$$

Now, we can express the difference $D^{*}\left(V_{0}\right)$ as $D^{*}\left(V_{0}\right)=2^{1-2 s} \pi^{-s} e\left[-\frac{s}{4}\right]\left(\Phi_{0}+\Gamma(s) \Omega_{0}\right)+2^{1-s} \Psi_{0}$.
Using the integral representation (35) of $L\left(\alpha, s,\left(p^{\prime}, q^{\prime}\right), c, d\right)$, we calculate $\Phi_{0}$. Therefore,

$$
\begin{aligned}
(1-e[s]) \Phi_{0}= & -\int_{I(\lambda, \infty)} t^{s-1} \frac{\exp (-(1 / 4+\alpha) t)+\exp (-(1 / 4+\alpha / 2) t)}{(1-\exp (-t))(1-\exp (-\alpha t))} \mathrm{d} t \\
& +e\left[\frac{s}{2}\right] \int_{I(\lambda, \infty)} t^{s-1} \frac{\exp (-(3 / 4+\alpha) t)+\exp (-(3 / 4+\alpha / 2) t)}{(1-\exp (-t))(1-\exp (-\alpha t))} \mathrm{d} t \\
& +2^{s-1}\left(1-e\left[\frac{s}{2}\right]\right) \int_{I(\lambda, \infty)} t^{s-1} \frac{\exp (-(1 / 2+\alpha) t)}{(1-\exp (-t))(1-\exp (-\alpha t))} \mathrm{d} t .
\end{aligned}
$$


Combining the first two integrals, we have

where

$$
\begin{aligned}
(1-e[s]) \Phi_{0}= & I_{0}+2^{s-1}\left(1-e\left[\frac{s}{2}\right]\right) \int_{I(\lambda, \infty)} t^{s-1} \\
& \frac{\exp (-(1 / 2+\alpha) t)}{(1-\exp (-t))(1-\exp (-\alpha t))} \mathrm{d} t,
\end{aligned}
$$

$$
I_{0}=\int_{I(\lambda, \infty)} t^{s-1} \frac{-\exp (-t / 4)(1-\exp (\pi i s-t / 2))(\exp (-\alpha t)+\exp (-\alpha t / 2))}{(1-\exp (-t))(1-\exp (-\alpha t))} \mathrm{d} t
$$

Now, making the change of variable $t \longleftrightarrow 2 t$, we get

$$
I_{0}=2^{s} \int_{I(\lambda, \infty)} t^{s-1} \frac{-\exp (-t / 2)(1-\exp (\pi i s-t))(\exp (-2 \alpha t)+\exp (-\alpha t))}{(1+\exp (-t))(1-\exp (-t))(1+\exp (-\alpha t))(1-\exp (-\alpha t))} \mathrm{d} t .
$$

Hence, after eliminating the common factor, we arrive at

Therefore,

$$
\begin{array}{r}
\begin{array}{r}
(1-e[s]) \Phi_{0}=2^{s} \int_{I(\lambda, \infty)} t^{s-1} \frac{\exp (-t / 2)(-1+\exp (\pi i s-t)) \exp (-\alpha t)}{(1+\exp (-t))(1-\exp (-t))(1-\exp (-\alpha t))} \mathrm{d} t \\
+2^{s-1}\left(1-e\left[\frac{s}{2}\right]\right) \int_{I(\lambda, \infty)} t^{s-1} \frac{\exp (-(1 / 2+\alpha) t)}{(1-\exp (-t))(1-\exp (-\alpha t))} \mathrm{d} t . \\
\Psi_{0}=\eta\left(\alpha, s, 1,-\frac{1}{4}\right)-\eta\left(\alpha, s, \frac{1}{4}, 0\right)+\eta\left(\alpha, s, \frac{1}{2},-\frac{1}{4}\right) \\
\text { Therefore, } \\
\Phi_{0}=-\frac{2^{s-1}}{1-e[s / 2]} \int_{I(\lambda, \infty)} t^{s-1} \frac{\exp (-(1 / 2+\alpha) t)}{(1+\exp (-t))(1-\exp (-\alpha t))} \mathrm{d} t . \\
-\eta\left(\alpha, s, \frac{1}{4}, \frac{1}{2}\right)-2^{s}\left\{\eta\left(\alpha, s, 1, \frac{1}{2}\right)-\eta\left(\alpha, s, \frac{1}{2}, 0\right)\right\} .
\end{array}
\end{array}
$$

Our next target is to calculate $\Psi_{0}$. Using (23), we have

Now, using the definition of the $\eta$-function, we get

$$
\begin{aligned}
\Psi_{0}= & \sum_{n=1}^{\infty} n^{s-1} \frac{e[n(\alpha-1 / 4)]}{1-e[n \alpha]}+\sum_{n=1}^{\infty} n^{s-1} \frac{e[n(\alpha / 2-1 / 4)]}{1-e[n \alpha]}-\sum_{n=1}^{\infty} n^{s-1} \frac{e[n \alpha / 4]}{1-e[n \alpha]}-\sum_{n=1}^{\infty} n^{s-1} \frac{e[n(\alpha / 4+1 / 2)]}{1-e[n \alpha]} \\
& -2^{s} \sum_{n=1}^{\infty} n^{s-1} \frac{e[n(\alpha+1 / 2)]}{1-e[n \alpha]}+2^{s} \sum_{n=1}^{\infty} n^{s-1} \frac{e[n \alpha / 2]}{1-e[n \alpha]} \\
= & \sum_{n=1}^{\infty} n^{s-1} \frac{e[n(\alpha / 2-1 / 4)](e[n \alpha / 2]+1)}{1-e[n \alpha]}-2 \sum_{n=1}^{\infty} \frac{(2 n)^{s-1} \frac{e[2 n(\alpha / 2-1 / 4)](1+e[n \alpha])}{1-e[2 n \alpha]}}{1-e[n \alpha]}+2 \sum_{n=1}^{\infty}(2 n)^{s-1} \frac{e[2 n \alpha / 4](1+e[n \alpha])}{1-e[2 n \alpha]} \\
& -\sum_{n=1}^{\infty} n^{s-1} \frac{e[n \alpha / 4]\left(1+(-1)^{n}\right)}{1-e[n \alpha]} \\
= & -\sum_{n=1}^{\infty} n^{s-1} \frac{e[n(\alpha / 2+1 / 4)](e[n \alpha / 2]+1)}{1-2 \sum_{n=1}^{\infty}(2 n)^{s-1} \frac{e[3 n \alpha / 2]}{1-e[2 n \alpha]} .}
\end{aligned}
$$


To calculate $\Omega_{0}$, we use $2^{s} \zeta(s,(1 / 2))=\zeta(s,(1 / 4))+$ $\zeta(s,(3 / 4))$, and we get

$$
\Omega_{0}=\left(\zeta\left(s, \frac{1}{4}\right)-2^{s-1} \zeta\left(s, \frac{1}{2}\right)\right)=2^{-1}\left(\zeta\left(s, \frac{1}{4}\right)-\zeta\left(s, \frac{3}{4}\right)\right) .
$$

Finally, combining the expressions for $\Phi_{0}, \Psi_{0}$, and $\Omega_{0}$ we deduce Theorem 7 (60).

\section{Proof of Theorem $7(61)$}

By using Proposition 2 and from (42), we have

$$
\begin{aligned}
D^{*}\left(V_{1}\right)= & D^{*}\left(V_{1}, \alpha, s, \frac{1}{2}, 0\right) \\
= & (\alpha+1)^{-s} A^{*}\left(\frac{\alpha}{\alpha+1}, s, \frac{1}{2}, 0\right)-A^{*}\left(\alpha, s, \frac{1}{2}, 0\right) \\
= & \frac{2^{1-s}}{1-e[s]}(2 \pi)^{-s}\left\{e\left[-\frac{s}{4}\right] L\left(\alpha, s,\left(\frac{1}{4}, 0\right), 1,1\right)-e\left[\frac{s}{4}\right] L\left(\alpha, s,\left(-\frac{1}{4}, 0\right), 1,1\right)\right\} \\
& +\frac{2^{1-s}}{1-e[s]}(2 \pi)^{-s}\left\{e\left[-\frac{s}{4}\right] L\left(\alpha, s,\left(\frac{3}{4}, \frac{1}{2}\right), 1,1\right)-e\left[\frac{s}{4}\right] L\left(\alpha, s,\left(-\frac{3}{4},-\frac{1}{2}\right), 1,1\right)\right\} \\
& +\frac{2^{1-s}}{1-e[s]}\left\{H\left(\alpha, s,\left(\frac{3}{4}, \frac{1}{2}\right)\right)-H\left(\alpha, s,\left(\frac{1}{4}, \frac{1}{2}\right)\right)\right\} \\
& -\frac{2^{1-s} e[s / 2]}{1-e[s]}\left\{H\left(\alpha, s,\left(-\frac{3}{4},-\frac{1}{2}\right)\right)-H\left(\alpha, s,\left(-\frac{1}{4},-\frac{1}{2}\right)\right)\right\} \\
& -\frac{1-e[s / 2]}{1-e[s]}(2 \pi)^{-s} e\left[-\frac{s}{4}\right] L\left(\alpha, s,\left(\frac{1}{2}, 0\right), 1,1\right) .
\end{aligned}
$$

Let

$$
\begin{aligned}
(1-e[s]) \Phi_{1}= & L\left(\alpha, s,\left(\frac{1}{4}, 0\right), 1,1\right)+L\left(\alpha, s,\left(\frac{3}{4}, \frac{1}{2}\right), 1,1\right) \\
& -e\left[\frac{s}{2}\right] L\left(\alpha, s,\left(-\frac{1}{4}, 0\right), 1,1\right) \\
& -e\left[\frac{s}{2}\right] L\left(\alpha, s,\left(-\frac{3}{4},-\frac{1}{2}\right), 1,1\right) \\
& -\left(1-e\left[\frac{s}{2}\right]\right) 2^{s-1} L\left(\alpha, s,\left(\frac{1}{2}, 0\right), 1,1\right) .
\end{aligned}
$$

$$
\begin{aligned}
(1-e[s]) \Psi_{1}= & H\left(\alpha, s,\left(\frac{3}{4}, \frac{1}{2}\right)\right)-H\left(\alpha, s,\left(\frac{1}{4}, \frac{1}{2}\right)\right) \\
& -e\left[\frac{s}{2}\right] H\left(\alpha, s,\left(-\frac{3}{4},-\frac{1}{2}\right)\right) \\
& +e\left[\frac{s}{2}\right] H\left(\alpha, s,\left(-\frac{1}{4},-\frac{1}{2}\right)\right) .
\end{aligned}
$$

We now express (102) as

$$
D^{*}\left(V_{1}\right)=2^{1-2 s} \pi^{-s} e\left[-\frac{s}{4}\right] \Phi_{1}+2^{1-s} \Psi_{1} .
$$

Now, utilizing the integral representation (35) of $L\left(\alpha, s,\left(p^{\prime}, q^{\prime}\right), c, d\right)$, we have 


$$
\begin{aligned}
(1- & e[s]) \Phi_{1} \\
= & -\int_{I(\lambda, \infty)} t^{s-1} \frac{\exp (-(1 / 4+3(\alpha+1) / 4) t)+\exp (-(1 / 4+(\alpha+1) / 4) t)}{(1-\exp (-t))(1-\exp (-(\alpha+1) t))} \mathrm{d} t \\
& +e\left[\frac{s}{2}\right] \int_{I(\lambda, \infty)} t^{s-1} \frac{\exp (-(3 / 4+(\alpha+1) / 4) t)+\exp (-(3 / 4+3(\alpha+1) / 4) t)}{(1-\exp (-t))(1-\exp (-(\alpha+1) t))} \mathrm{d} t \\
& +2^{s-1}\left(1-e\left[\frac{s}{2}\right]\right) \int_{I(\lambda, \infty)} t^{s-1} \frac{\exp (-(1 / 2+(\alpha+1) / 2) t)}{(1-\exp (-t))(1-\exp (-(\alpha+1) t))} \mathrm{d} t .
\end{aligned}
$$

Again, we write the left hand side of the above equation as

$$
\begin{aligned}
(1-e[s]) \Phi_{1}= & I_{1} \\
& +2^{s-1}\left(1-e\left[\frac{s}{2}\right]\right) \int_{I(\lambda, \infty)} t^{s-1} \frac{\exp (-(1 / 2+(\alpha+1) / 2) t)}{(1-\exp (-t))(1-\exp (-(\alpha+1) t))} \mathrm{d} t,
\end{aligned}
$$

where

$$
I_{1}=\int_{I(\lambda, \infty)} t^{s-1} \frac{(-\exp (-t / 4)+\exp (\pi i s-(-3 t / 4)))(\exp (-(\alpha+1) t / 4)+\exp (-3(\alpha+1) t / 4))}{(1-\exp (-t))(1-\exp (-(\alpha+1) t))} \mathrm{d} t
$$

Now, by change of variable $t \longleftrightarrow 2 t$ followed by the elimination of the common factor $1+\exp (-(\alpha+1) t)$, we get

$$
I_{1}=2^{s} \int_{I(\lambda, \infty)} t^{s-1} \frac{\exp (-t / 2)(-1+\exp (\pi i s-t)) \exp (-(\alpha+1) t / 2)}{(1+\exp (-t))(1-\exp (-t))(1-\exp (-(\alpha+1) t))} \mathrm{d} t
$$

Thus, substituting $I_{1}$ in (107), we see that

$$
\Phi_{1}=-\frac{2^{s-1}}{1-e[s / 2]} \int_{I(\lambda, \infty)} t^{s-1} \frac{\exp (-(1 / 2+(\alpha+1) / 2) t)}{(1+\exp (-t))(1-\exp (-(\alpha+1) t))} \mathrm{d} t
$$

Using the definition of $H$-function, from (104), we have

$$
n^{s-1} \frac{e[n \alpha / 4+1 / 2](e[n \alpha / 2]-1)}{1-e[n \alpha]}
$$

$$
\begin{aligned}
\Psi_{1}= & \eta\left(\alpha, s, \frac{3}{4}, \frac{1}{2}\right)-\eta\left(\alpha, s, \frac{1}{4}, \frac{1}{2}\right) \\
= & \sum_{n=1}^{\infty} n^{s-1} \frac{e[3 n \alpha / 4+1 / 2]}{1-e[n \alpha]} \\
& -\sum_{n=1}^{\infty} n^{s-1} \frac{e[n \alpha / 4+1 / 2]}{1-e[n \alpha]} .
\end{aligned}
$$

The $n$th summand is

from which we may eliminate the common factor $e[(1 / 2) n \alpha]-1$. Therefore,

$$
\Psi_{1}=\sum_{n=1}^{\infty} n^{s-1} \frac{(-1)^{n-1} e[n \alpha / 4]}{e[n \alpha / 2]+1}=\frac{1}{2} \sum_{n=1}^{\infty} n^{s-1} \frac{(-1)^{n-1}}{\cos (\pi n \alpha / 2)} .
$$

Now, we substitute (110) and (113) in (102) and finally get 


$$
\begin{aligned}
(\alpha+1)^{-s} A^{*}\left(\frac{\alpha}{\alpha+1}, s, \frac{1}{2}, 0\right)-A^{*}\left(\alpha, s, \frac{1}{2}, 0\right) \\
=-\frac{(2 \pi)^{-s} e[-s / 4]}{(1-e[s / 2])} \int_{I(\lambda, \infty)} t^{s-1} \frac{\exp (-t / 2)}{(1+\exp (-t))} \frac{\exp (-(\alpha+1) t / 2)}{(1-\exp (-(\alpha+1) t))} \mathrm{d} t \\
\quad+2^{-s} \sum_{n=1}^{\infty} n^{s-1} \frac{(-1)^{n-1}}{\cos (\pi \alpha n / 2)}
\end{aligned}
$$

This completes the proof of Theorem 7 (61).

\section{Proof of Theorem 7 (62)}

We follow the same route: first, we use Proposition 2 and then using (43), we obtain

$$
\begin{aligned}
D^{*}\left(V_{2}\right)= & D^{*}\left(V_{2}, \alpha, s, \frac{1}{2}, 0\right) \\
= & (\alpha-1)^{-s} A^{*}\left(\frac{-\alpha}{\alpha-1}, s, \frac{1}{2}, 0\right)-A^{*}\left(\alpha, s, \frac{1}{2}, 0\right) \\
= & \frac{2^{1-s}}{1-e[s]}(2 \pi)^{-s}\left\{e\left[-\frac{s}{4}\right] L\left(\alpha, s,\left(-\frac{1}{4}, 0\right), 1,-1\right)-e\left[\frac{s}{4}\right] L\left(\alpha, s,\left(\frac{1}{4}, 0\right), 1,-1\right)\right\} \\
& +\frac{2^{1-s}}{1-e[s]}(2 \pi)^{-s}\left\{e\left[-\frac{s}{4}\right] L\left(\alpha, s,\left(\frac{1}{4},-\frac{1}{2}\right), 1,-1\right)-e\left[\frac{s}{4}\right] L\left(\alpha, s,\left(-\frac{1}{4}, \frac{1}{2}\right), 1,-1\right)\right\} \\
& -\frac{1-e[s / 2]}{1-e[s]}(2 \pi)^{-s} e\left[-\frac{s}{4}\right] L\left(\alpha, s,\left(-\frac{1}{2}, 0\right), 1,-1\right) \\
& +\frac{2^{1-s}}{1-e[s]}\left\{H\left(\alpha, s,\left(-\frac{1}{4}, 0\right)\right)-H\left(\alpha, s,\left(\frac{1}{4}, 0\right)\right)\right\} \\
& -\frac{2^{1-s} e[s / 2]}{1-e[s]}\left\{H\left(\alpha, s,\left(\frac{1}{4}, 0\right)\right)-H\left(\alpha, s,\left(-\frac{1}{4}, 0\right)\right)\right\} \\
= & 2^{1-2 s} \pi^{-s} e\left[-\frac{s}{4}\right] \Phi_{2}+2^{1-s} \Psi_{2},
\end{aligned}
$$

where

$$
\begin{aligned}
(1-e[s]) \Phi_{2}= & L\left(\alpha, s,\left(-\frac{1}{4}, 0\right), 1,-1\right)-e\left[\frac{s}{2}\right] L\left(\alpha, s,\left(\frac{1}{4}, 0\right), 1,-1\right) \\
& +L\left(\alpha, s,\left(\frac{1}{4},-\frac{1}{2}\right), 1,-1\right)-e\left[\frac{s}{2}\right] L\left(\alpha, s,\left(-\frac{1}{4}, \frac{1}{2}\right), 1,-1\right) \\
& -\left(1-e\left[\frac{s}{2}\right]\right) 2^{s-1} L\left(\alpha, s,\left(-\frac{1}{2}, 0\right), 1,-1\right), \\
(1-e[s]) \Psi_{2}= & H\left(\alpha, s,\left(-\frac{1}{4}, 0\right)\right)-H\left(\alpha, s,\left(\frac{1}{4}, 0\right)\right) \\
& -e\left[\frac{s}{2}\right] H\left(\alpha, s,\left(\frac{1}{4}, 0\right)\right)+e\left[\frac{s}{2}\right] H\left(\alpha, s,\left(-\frac{1}{4}, 0\right)\right) \\
= & \left(1+e\left[\frac{s}{2}\right]\right)\left(H\left(\alpha, s,\left(-\frac{1}{4}, 0\right)\right)-H\left(\alpha, s,\left(\frac{1}{4}, 0\right)\right)\right) .
\end{aligned}
$$


To simplify $\Phi_{2}$, we make use of the integral representation (35) of $L\left(\alpha, s,\left(p^{\prime}, q^{\prime}\right), c, d\right)$. So, we have

$$
\begin{aligned}
(1-e[s]) \Phi_{2}= & I_{2} \\
& +2^{s-1}\left(1-e\left[\frac{s}{2}\right]\right) \int_{I(\lambda, \infty)} t^{s-1} \frac{\exp (-(1 / 2+(\alpha-1) / 2) t)}{(1-\exp (-t))(1-\exp (-(\alpha-1) t))} \mathrm{d} t
\end{aligned}
$$

where

$$
I_{2}=\int_{I(\lambda, \infty)} t^{s-1} \frac{(-\exp (-t / 4)+\exp (\pi i s-3 t / 4))(\exp -(\alpha-1) t / 4+\exp (-3(\alpha-1) t / 4))}{(1-\exp (-t))(1-\exp (-(\alpha-1) t))} \mathrm{d} t
$$

As before, by eliminating the common factor $1-\exp (-t)$, we obtain

$$
I_{2}=2^{s} \int_{I(\lambda, \infty)} t^{s-1} \frac{\exp (-t / 2) \exp (-(\alpha-1) t / 2)(-1+\exp (\pi i s-t))}{(1+\exp (-t))(1-\exp (-t))(1-\exp (-(\alpha-1) t))} \mathrm{d} t
$$

Whence, it follows that

$$
\Phi_{2}=-\frac{2^{s-1}}{1-e[s / 2]} \int_{I(\lambda, \infty)} t^{s-1} \frac{\exp (-(1 / 2+(\alpha-1) / 2) t)}{(1+\exp (-t))(1-\exp (-(\alpha-1) t))} \mathrm{d} t
$$

While handling (117), we decompose it as

$$
\Psi_{2}=-\sum_{n=1}^{\infty} n^{s-1} \frac{e[n \alpha / 4]}{e[n \alpha / 2]+1}=-\frac{1}{2} \sum_{n=1}^{\infty} n^{s-1} \frac{1}{\cos (\pi n \alpha / 2)} .
$$

$$
\Psi_{2}=\eta\left(\alpha, s, \frac{3}{4}, 0\right)-\eta\left(\alpha, s, \frac{1}{4}, 0\right) .
$$

In the series expression of $\Psi_{2}$, we factor out $e[n \alpha / 4]$ as before and eliminate the common factor $(e[n \alpha / 2]-1)$ to have

Finally, substituting the expressions for $\Phi_{2}$ and $\Psi_{2}$, we obtain

$$
\begin{aligned}
(\alpha-1)^{-s} A^{*}\left(\frac{-\alpha}{\alpha-1}, s, \frac{1}{2}, 0\right)-A^{*}\left(\alpha, s, \frac{1}{2}, 0\right) \\
=-\frac{(2 \pi)^{-s} e[-s / 4]}{1-e[(s / 2)]} \int_{I(\lambda, \infty)} t^{s-1} \frac{\exp (-t / 2)}{(1+\exp (-t))} \frac{\exp (-(\alpha-1) t / 2)}{(1-\exp (-(\alpha-1)) t)} \mathrm{d} t \\
\quad-2^{-s} \sum_{n=1}^{\infty} n^{s-1} \frac{1}{\cos (\pi n \alpha / 2)} .
\end{aligned}
$$


This finishes the proof of Theorem 7 (62).

\section{Proof of Corollary 1}

We conclude this chapter by finally proving Corollary 1 . We add (114) and (124) and derive that

$$
\begin{aligned}
& (\alpha+1)^{-s} A^{*}\left(\frac{\alpha}{\alpha+1}, s, \frac{1}{2}, 0\right)+(\alpha-1)^{-s} A^{*}\left(\frac{-\alpha}{\alpha-1}, s, \frac{1}{2}, 0\right)-2 A^{*}\left(\alpha, s, \frac{1}{2}, 0\right) \\
& =-\frac{(2 \pi)^{-s} e[-s / 4]}{1-e[s / 2]} \int_{I(\lambda, \infty)} t^{s-1} \frac{\exp (-t / 2)}{(1+\exp (-t))} \frac{\exp (-(\alpha+1) t / 2)}{(1-\exp (-(\alpha+1) t))} \mathrm{d} t \\
& -\frac{(2 \pi)^{-s} e[-s / 4]}{1-e[-s / 2]} \int_{I(\lambda, \infty)} t^{s-1} \frac{\exp (-t / 2)}{(1+\exp (-t))} \frac{\exp (-(\alpha-1) t / 2)}{(1-\exp (-(\alpha-1) t))} \mathrm{d} t \\
& +2^{-s} \sum_{n=1}^{\infty} n^{s-1} \frac{(-1)^{n-1}}{\cos (\pi n \alpha / 2)}-2^{-s} \sum_{n=1}^{\infty} n^{s-1} \frac{1}{\cos (\pi n \alpha / 2)} \\
& =-\frac{(2 \pi)^{-s} e[-s / 4]}{1-e[-s / 2]} \int_{I(\lambda, \infty)} t^{s-1} \frac{\exp (-t / 2)}{1+\exp (-t)}\left\{\frac{\exp (-(\alpha+1) t / 2)}{1-\exp (-(\alpha+1) t)}+\frac{\exp (-(\alpha+1) t / 2)}{1-\exp (-(\alpha-1) t)}\right\} \mathrm{d} t \\
& -2 \cdot 2^{-s} \sum_{n=1}^{\infty}(2 n)^{s-1} \frac{1}{\cos (2 \pi n \alpha / 2)}
\end{aligned}
$$

Now, in the above expression, $2 A^{*}(\alpha, s, 1 / 2,0)$ on the left hand side and secant zeta function on the right hand side will cancel each other, as they are the same (from (50)). Therefore, we have

$$
\begin{aligned}
(\alpha+1)^{-s} A^{*}\left(\frac{\alpha}{\alpha+1}, s, \frac{1}{2}, 0\right)+(\alpha-1)^{-s} A^{*}\left(\frac{-\alpha}{\alpha-1}, s, \frac{1}{2}, 0\right) \\
=-\frac{(2 \pi)^{-s} e[-(s / 4)]}{1-e[s / 2]} \int_{I(\lambda, \infty)} t^{s-1} \frac{\exp (-(1 / 2) t)}{1+\exp (-t)} \\
\cdot\left\{\frac{\exp (-(1 / 2)(\alpha+1) t)}{1-\exp (-(\alpha+1) t)}+\frac{\exp (-(1 / 2)(\alpha-1) t)}{1-\exp (-(\alpha-1) t)}\right\} \mathrm{d} t \\
=-\frac{(2 \pi)^{-s} e[-(s / 4)]}{1-e[s / 2]} \int_{I(\lambda, \infty)} t^{s-1} \sum_{m=0}^{\infty} E_{m}\left(\frac{1}{2}\right) \frac{t^{m}}{2 m !},
\end{aligned}
$$

and thus Corollary 1 follows.

\section{Future Work}

By the virtue of the work of Lewittes, Berndt, and Arakawa, it would be interesting to find the general modular transformation formula for $A^{*}(\alpha, s, p, q)$ for all $(p, q) \in \mathbb{R}^{2}$ and from which one would like to see the truth of our Conjecture 2.

\section{Data Availability}

No data were used to support this study.

\section{Conflicts of Interest}

The authors declare that they have no conflicts of interest.

\section{Acknowledgments}

B. Maji wishes to thank the Indian Institute of Technology Indore for the conductive environment.

\section{References}

[1] B. Riemann, Fragmente Über Die Grenzfälle der Ellipitischen Modulfunctionen, Collected Works of Bernhard Riemann, H. Weber, Ed., pp. 455-465, Dover, New York, NY, USA, 2nd edition, 1852.

[2] A. Wintner, “On Riemann's fragment concerning elliptic modular functions," American Journal of Mathematics, vol. 63, no. 3, pp. 628-634, 1941.

[3] A. D. Reyna, "Riemann's fragment on the limit values of elliptic modular functions," The Ramanujan Journal, vol. 8, pp. 57-123, 2004.

[4] N. Wang, "On Riemann's posthumous fragment II on the limit values of elliptic modular functions," The Ramanujan Journal, vol. 24, no. 2, pp. 129-145, 2011.

[5] J. Lewittes, "Analytic continuation of Eisenstein series," Transactions of the American Mathematical Society, vol. 171, p. 469, 1972.

[6] B. C. Berndt, "Generalized Dedekind eta-functions and generalized Dedekind sums," Transactions of the American Mathematical Society, vol. 178, pp. 495-508, 1973.

[7] T. Arakawa, "Generalized eta-functions and certain ray class invariants of real quadratic fields," Mathematische Annalen, vol. 260, no. 4, pp. 475-494, 1982. 
[8] T. Arakawa, "Dirichlet series $\sum_{n=1}^{\infty} \cot \pi n \alpha / n^{s}$ Dedekind sums and Hecke L- functions for real quadratic fields," Commentarii-Mathematici-Universitatis-Sancti-Pauli, vol. 37, pp. 209-235, 1988.

[9] M. Lerch, "Sur une série analogue aux fonctions modulaires," Comptes rendus de l'Académie des Sciences, vol. 138, pp. 952-954, 1904.

[10] B. C. Berndt, "Dedekind sums and a paper of G. H. Hardy," Journal of the London Mathematical Society, vol. s2-13, no. 1, pp. 129-137, 1976.

[11] M. Lalín, F. Rodrigue, and M. Rogers, "Secant zeta functions," Journal of Mathematical Analysis and Applications, vol. 409, no. 1, pp. 197-204, 2014.

[12] B. C. Berndt and A. Straub, "On a secant Dirichlet series and Eichler integrals of Eisenstein series," Mathematische Zeitschrift, vol. 284, no. 3-4, pp. 827-852, 2016.

[13] P. Charollais and M. Greenberg, "Rationality of secant zeta values," Annales Mathématiques du Québec, vol. 38, no. 1, pp. 1-6, 2014. 